\title{
Spatially resolved spectroscopy of Coma cluster early-type galaxies
}

\section{The stellar population gradients ${ }^{\star}$}

\author{
D. Mehlert ${ }^{1}$, D. Thomas ${ }^{2}$, R. P. Saglia ${ }^{3}$, R. Bender ${ }^{2,3}$, and G. Wegner ${ }^{4}$ \\ 1 Landessternwarte Heidelberg, Königstuhl, 69117 Heidelberg, Germany \\ 2 Max-Planck-Institut für extraterrestrische Physik, Giessenbachstraße, 85748 Garching, Germany \\ 3 Universitäts-Sternwarte München, Scheinerstraße 1, 81679 München, Germany \\ ${ }^{4}$ Department of Physics and Astronomy, 6127 Wilder Laboratory, Dartmouth College, Hanover, NH 03755-3528, USA
}

Received 7 March 2003 / Accepted 27 May 2003

\begin{abstract}
Based on Paper I of this series (Mehlert et al. 2000), we derive central values and logarithmic gradients for the $\mathrm{H} \beta$, $\mathrm{Mg}$ and $\mathrm{Fe}$ indices of 35 early-type galaxies in the Coma cluster. We find that pure elliptical galaxies have on average slightly higher velocity dispersions, lower $\mathrm{H} \beta$, and higher metallic line-strengths than galaxies with disks (S0). The latter form two families, one comparable to the ellipticals and a second one with significantly higher $\mathrm{H} \beta$, and weaker metallic lines. Our measured logarithmic gradients within the effective radius are $\langle\Delta \mathrm{Mg} b\rangle \approx-0.037,\langle\Delta\langle\mathrm{Fe}\rangle\rangle \approx-0.029,\langle\Delta \mathrm{H} \beta\rangle \approx+0.017$ and $\langle\Delta \sigma\rangle \approx-0.063$. The gradients strongly correlate with the gradients of $\sigma$, but only weakly with the central index values and galaxy velocity dispersion.

Using stellar population models with variable element abundance ratios from Thomas et al. (2003a) we derive average ages, metallicities and $[\alpha / \mathrm{Fe}]$ ratios in the center and at the effective radius. We find that the $\alpha / \mathrm{Fe}$ ratio correlates with velocity dispersion and drives $30 \%$ of the $\mathrm{Mg}-\sigma$ relation, the remaining $70 \%$ being caused by metallicity variations. We confirm previous findings that part of the lenticular galaxies in the Coma cluster host very young ( $\sim 2 \mathrm{Gyr}$ ) stellar populations, hence must have experienced relatively recent star formation episodes. Again in accordance with previous work we derive negative metallicity gradients ( -0.16 dex per decade) that are significantly flatter than what is expected from gaseous monolithic collapse models, pointing to the importance of mergers in the galaxy formation history. Moreover, the metallicity gradients correlate with the velocity dispersion gradients, confirming empirically earlier suggestions that the metallicity gradient in ellipticals is produced by the local potential well. The gradients in age are negligible, implying that no significant residual star formation has occurred either in the center or in the outer parts of the galaxies, and that the stellar populations at different radii must have formed at a common epoch. For the first time we derive the gradients of the $\alpha / \mathrm{Fe}$ ratio and find them very small on average. Hence, $\alpha / \mathrm{Fe}$ enhancement is not restricted to galaxy centers but it is a global phenomenon. Our results imply that the $\mathrm{Mg}-\sigma$ local relation inside a galaxy, unlike the global $\mathrm{Mg}-\sigma$ relation, must be primarily driven by metallicity variations alone. Finally we note that none of the stellar population parameters or their gradients depend on the density profile of the Coma cluster, even though it spans 3 dex in density.
\end{abstract}

Key words. galaxies: clusters: individual: Coma - galaxies: elliptical and lenticular, cD - galaxies: stellar content

\section{Introduction}

This is the third paper of a series studying the dynamics and the stellar populations of a sample of 35 early-type galaxies in the Coma cluster, the richest of the local universe and therefore an ideal place to test the theories of galaxy formation as a function of environmental density. In Mehlert et al. (2000, hereafter Paper I) we presented the photometry and the long-slit

Send offprint requests to: D. Mehlert, e-mail: dmehlert@lsw.uni-heidelberg.de

* Appendix A (Tables A.1-A.3) is only available in electronic form at http://www.edpsciences.org spectroscopy along the major axis of the galaxies. In Wegner et al. (2002) this dataset is complemented by long-slit spectroscopy along the minor axis and parallel to the major axis for 10 objects of the sample. Here we focus on the radial profiles of the $\mathrm{Mg}$, Fe and $\mathrm{H} \beta$ indices derived in Paper I, exploiting newly developed stellar population models (Thomas et al. 2003a, hereafter TMB) to study the metallicity, the age and the $\alpha / \mathrm{Fe}$ distributions inside the galaxies and relate these findings to the global relations among the galaxies.

Recently, a number of papers have investigated the constraints on the ages, metallicities and element abundances of Coma cluster galaxies coming from (central) colors and 
spectroscopic (Lick) index measurements and the possible influence of the environmental density. Jørgensen (1999) studies $115 \mathrm{E}$ and $\mathrm{S} 0$ galaxies in the central region of the cluster, suggesting that metallicities are strongly anti-correlated with the mean ages of the galaxies. Poggianti et al. (2001a) analyze the spectra of 52 early-type Coma galaxies and find that more than $40 \%$ of the S0s are found to have undergone star formation in their central regions during the last $5 \mathrm{Gyr}$, while such activity seems absent in ellipticals (see also Trager et al. 2003). Poggianti et al. (2001b) extend this study to a sample of 257 galaxies with no emission lines, spanning a wide magnitude range. They find that age and metallicity are anticorrelated in any given luminosity bin and that in the central regions of the cluster a large fraction of galaxies at any luminosity shows no evidence for star formation occurring in their central regions at redshift $z<2$. Carter et al. (2002) focus on the possible environmental dependence of the indices, finding that after allowing for the correlation with magnitude, galaxies near the core of the cluster have stronger $\mathrm{Mg}_{2}$, while the $\langle\mathrm{Fe}\rangle$ and $\mathrm{H} \beta$ values show a much weaker sensitivity to the cluster distance. In contrast, Kuntschner et al. (2002) study a sample of "field" E/S0 galaxies in low-density environments, reaching the conclusion that these objects are younger than E/S0s in clusters (by $\approx 2-3 \mathrm{Gyr}$ ) and more metal-rich (by $\approx 0.2 \mathrm{dex}$ ). They confirm that an anti-correlation of age and metallicity is responsible for maintaining the zero-point of the $\mathrm{Mg}-\sigma$ relation (see also Kuntschner et al. 2001, pointing out the role of correlated errors in generating this anti-correlation).

Similar to Terlevich \& Forbes (2002), these studies break the metallicity-age degeneracy by combining pairs of indices with nearly orthogonal dependences on metallicity and age, and are based on simple stellar population models (Worthey 1994; Vazdekis et al. 1996). They take into account the overabundance of the $\alpha$-elements with ad-hoc assumptions (Jørgensen 1999) or simple, approximated recipes (Trager et al. 2000) and might be severely affected by systematics induced by complex metallicity distributions (Maraston \& Thomas 2000). However, they provide a way to test the low-redshift predictions of galaxy formation models and distinguish between the traditional "monolithic collapse" of proto-galactic gas clouds (Eggen et al. 1962; Jimenez et al. 1999) and the hierarchical models of Cold Dark Matter cosmologies (Kauffmann 1996; Baugh et al. 1996; Cole et al. 2000). Moreover, they allow a powerful insight in the mechanisms generating the global relations observed with the structure parameters of early-type galaxies (the color-magnitude relation, Bower et al. 1992; the $\mathrm{Mg}-\sigma$ relation, Bender et al. 1993; Colless et al. 1999; the Fundamental Plane, Djorgovski \& Davis 1987; Dressler et al. 1987) and regulating their scatter. In addition, they are the local counterpart of the studies of the evolution of early-type galaxies with redshift in clusters (van Dokkum \& Franx 1996; Bender et al. 1998; van Dokkum \& Standford 2003) and in the field (Treu et al. 1999; van Dokkum et al. 2001). Analyzing a large number of objects, they are also able to detect the possibly subtle effects of the environment.

The present paper cannot compete in terms of galaxy numbers with the literature discussed above. Rather, it relies on the high quality of the data and stresses two aspects neglected in the past: first a rigorous quantitative analysis of the $\alpha / \mathrm{Fe}$ role in determining the ages and metallicities of early-type galaxies, and second the discussion of the radial variations of the stellar populations of the galaxies. The former is based on newly developed models of the Lick indices (TMB) that incorporate the response functions of Tripicco \& Bell (1995), allowing a proper treatment of the element abundances. The latter provides an additional tool to test the predictions of galaxy formation models.

"Classical" monolithic collapse models with Salpeter IMF (Carlberg 1984) generate steep metallicity gradients (the centers are more metal rich than the outer parts), shallow positive age gradients (the centers are slightly younger than the outer parts) and positive overabundance gradients (the centers are less $\alpha /$ Fe enhanced, Thomas et al. 1999). The merger trees typical of hierarchical models of galaxy formation (Lacey \& Cole 1993) are expected to dilute gradients originally present in the merging galaxies and therefore give end-products with milder gradients (White 1980), although detailed quantitative predictions are still lacking. There is consensus that the radial changes in colors and line indices observed in local ellipticals reflect variation in metallicities rather than age (Faber 1977; Davies et al. 1993; Kobayashi \& Arimoto 1999), a conclusion confirmed by the study of the evolution of the color gradients with redshift (Saglia et al. 2000; Tamura et al. 2000). However, nothing is known about possible radial gradients of the $\alpha / \mathrm{Fe}$ abundance ratio detected at the centers of early-type galaxies. If no $\alpha / \mathrm{Fe}$ gradients are present, then the bulk of the stars of elliptical galaxies are $\alpha / \mathrm{Fe}$ overabundant and the constraint of short ( $\leq 1$ Gyr) formation time-scales (e.g., Matteucci 1994; Thomas et al. 1999) applies to the stellar populations of the galaxies as a whole, and not only to the galaxy centers. Our dataset clarifies this issue.

The paper is organized as follows. Section 2 describes the dataset. Section 3 discusses the central values and the radial profiles of the line indices. Section 4 derives constraints on the age, metallicities and the $[\alpha / \mathrm{Fe}]$ distributions inside the galaxies (central values and gradients). Conclusions are drawn in Sect. 5.

\section{The data sample}

The basis of this investigation are the radial line index profiles of $\mathrm{Mg} b,\langle\mathrm{Fe}\rangle$, and $\mathrm{H} \beta$ as well as the profile of the velocity dispersion $\sigma$ derived for 35 early-type galaxies in Mehlert et al. (2000, Paper I). These parameters were obtained from high $S / N$ and spatially resolved longslit spectra obtained along the major axis of the galaxies. Details on the galaxy sample, the observations, data reduction and parameter derivation are described in Paper I. With this dataset we can not only investigate the stellar population of the galaxies based on their central line indices, but also derive the gradients of the line indices and the stellar population parameters like age, metallicity and the $[\alpha / \mathrm{Fe}]$ ratios. Since the sample analyzed here comprises $\mathrm{E}$ and $\mathrm{S} 0$ galaxies and spans a range of 3 dex in local mass densities (as determined by X-ray observations, Briel et al. 1992), the influence of galaxy type and environmental density on the stellar population of the Coma cluster early type galaxies can be explored. In Paper I we already showed that our derived line 
index and $\sigma$ values agree quite well with literature data. This is also supported by a detailed comparison to a different galaxy sample in Moore et al. (2002).

\section{Line indices}

\subsection{Central values}

Table A.1 lists the central values of the indices $\mathrm{H} \beta, \operatorname{Mg} b$, Fe5270, Fe5335 and of the velocity dispersion $\sigma$ with the respective errors. The values have been derived from the major axis profiles shown in Paper I averaging data points inside $0.1 a_{\mathrm{e}}$, where $a_{\mathrm{e}}=r_{\mathrm{e}} / \sqrt{1-\epsilon}$ is the distance along the major axis, that corresponds to the effective radius $r_{\mathrm{e}}$ of the galaxy with ellipticity $\epsilon$. The central values were calculated by weighting proportional to the signal-to-noise ratio $S / N$ :

$\langle$ Index $\rangle=\frac{\sum_{i} \operatorname{Index}_{i} \times(S / N)_{i}}{\sum_{i}(S / N)_{i}}$,

where $i$ indicates the radial points available. This way of weighting our radial values turned out to be a good compromise to optimize the quality of the mean values without overemphazising the central value.

In the following we indicate the average iron index with $\langle\mathrm{Fe}\rangle=(\mathrm{Fe} 5270+\mathrm{Fe} 5335) / 2$ (Gorgas et al. 1990) and the combined magnesium-iron index with

$[\mathrm{MgFe}]^{\prime}=\sqrt{\mathrm{Mg} b(0.72 \times \mathrm{Fe} 5270+0.28 \times \mathrm{Fe} 5335)}$

newly defined by TMB. This index turns out to be completely independent of the $\alpha / \mathrm{Fe}$ and hence serves best as a metallicity tracer. The usual index $[\mathrm{MgFe}]=\sqrt{\mathrm{Mg} b\langle\mathrm{Fe}\rangle}$ earlier defined by González (1993) still depends a little on $\alpha / \mathrm{Fe}$ (see also Sect. 4.1). We also consider the $\mathrm{Mg} b^{\prime}$ index (see Colless et al. 1999):

$\operatorname{Mg} b^{\prime}=-2.5 \times \log \left(1-\frac{\operatorname{Mg} b}{\Delta \lambda}\right)$,

with $\Delta \lambda=32.25 \AA$.

In Fig. 1 we plot the central index values as a function of velocity dispersion. Our data show a tight $\mathrm{Mg}-\sigma$ correlation (panel a) in good agreement with the literature (e.g., Bender et al. 1993; Colless et al. 1999). Correlations of velocity dispersion with the other indices $\langle\mathrm{Fe}\rangle$ and $\mathrm{H} \beta$, instead, are much less well-defined (panels $\mathrm{b}$ and $\mathrm{c}$ ) in agreement with results from, e.g., Jørgensen et al. (1999). Still, $\langle\mathrm{Fe}\rangle$ seems to be weakly correlated with $\sigma$ (see also Kuntschner 2000). Linear least square fits (see the solid lines in Fig. 1) yield the following relations:

$\operatorname{Mg} b^{\prime}=-0.11( \pm 0.03)+0.12( \pm 0.01) \times \log \sigma$

$\log \mathrm{Mg} b=-0.04( \pm 0.08)+0.30( \pm 0.03) \times \log \sigma$

$\log \langle\mathrm{Fe}\rangle=0.13( \pm 0.11)+0.13( \pm 0.05) \times \log \sigma$.

Although there is no striking difference between elliptical and lenticular galaxies in Fig. 1 (see also Mehlert 1998), we note that the SOs in our sample have on average lower central velocity dispersions, and tend to have slightly lower metallic indices at a given $\sigma$. The most significant feature is the separation of the lenticulars in two distinct subclasses, one being

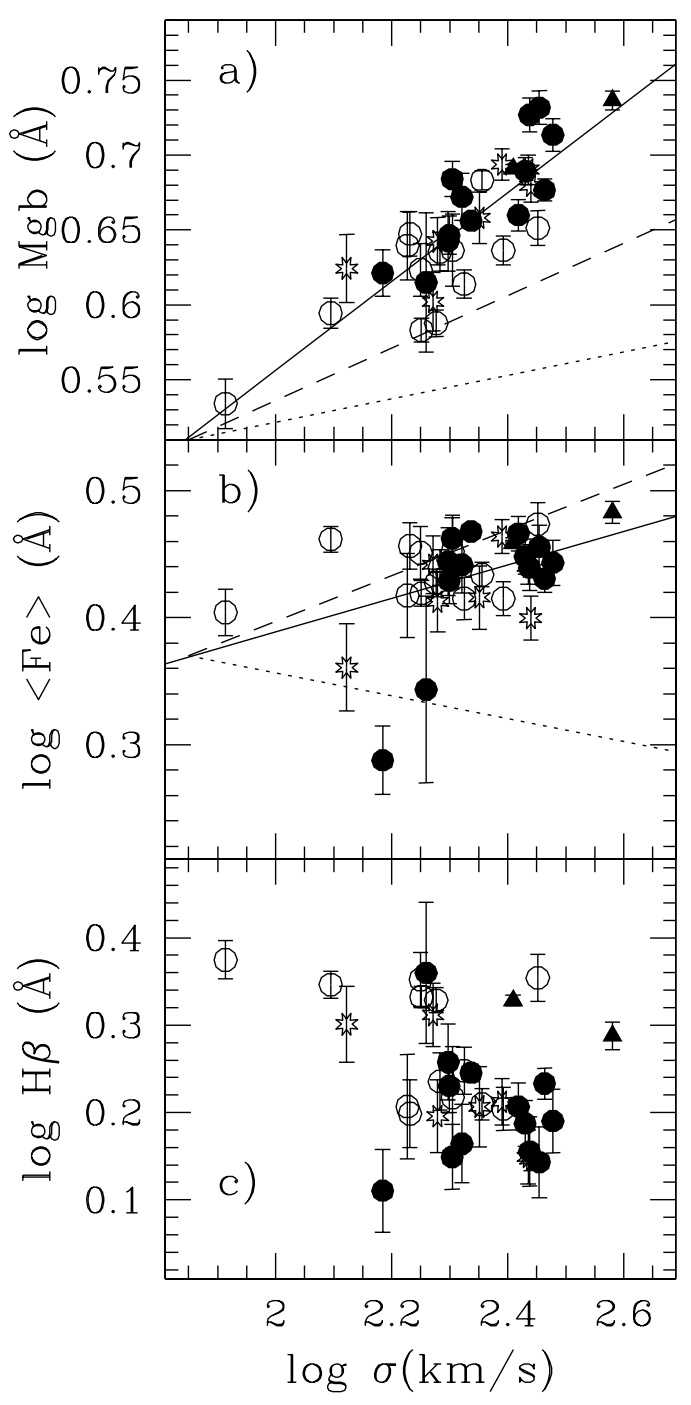

Fig. 1. The central line indices $\mathrm{Mg} b$ (panel a)), $\langle\mathrm{Fe}\rangle($ panel b)), and $\mathrm{H} \beta$ (panel c)) versus the velocity dispersion $\sigma$ for the 35 early type galaxies of the Coma cluster sample. Filled triangles indicate the $2 \mathrm{cDs}$, filled circles Es, open circles S0s and open stars E/S0s. The solid lines are linear least square fits (see text). The long and short dashed lines show the contributions to the $\mathrm{Mg}-\sigma$ and $\mathrm{Fe}-\sigma$ relations due to metallicity and $\alpha / \mathrm{Fe}$ ratio variations, respectively (see Sect. 4.2.2).

almost indistinguishable from the ellipticals, the other having extraordinarily high $\mathrm{H} \beta$ values. We will come back to this point in Sect. 4, where we discuss the derived ages and element abundances.

Note that also the two cD galaxies (triangles) have Balmer lines that are unusually strong given the relatively high galaxy's velocity dispersion. For a detailed discussion of the properties of the stellar populations (age, metallicity and $\alpha / \mathrm{Fe}$ ratios) derived from these indices see Sect. 4.2.

Finally it should be emphasized that we do not find any dependence of the derived indices or the galaxies velocity dispersion on the density profile of the Coma cluster. 

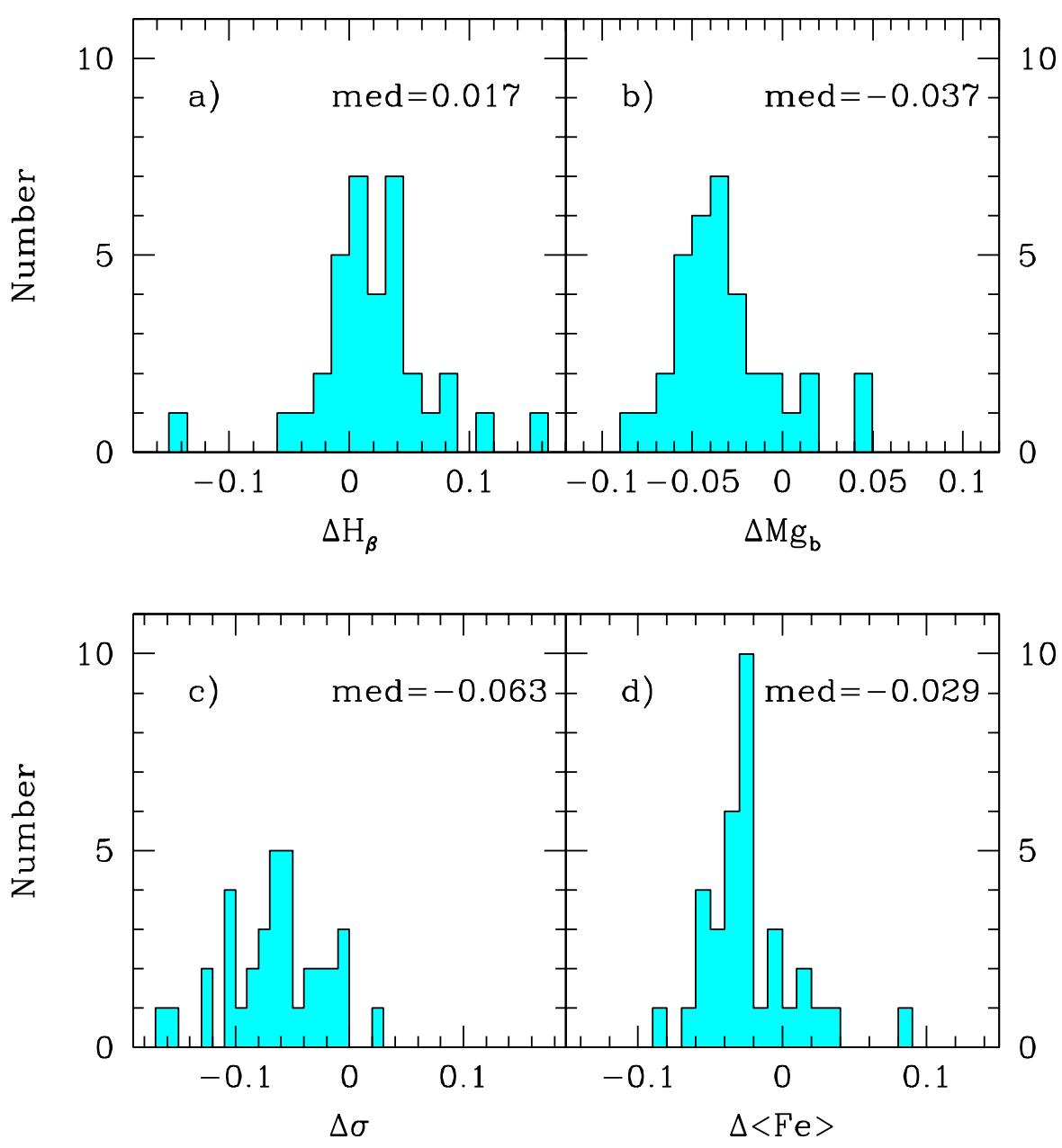

Fig. 2. Distributions of the fitted logarithmic gradients of the indices, $\mathrm{H} \beta(\mathbf{a}), \operatorname{Mg} b(\mathbf{b}),\langle\mathrm{Fe}\rangle(\mathbf{c})$, and the velocity dispersion $\sigma$ (d). The median of the gradients is indicated in the individual panels.

\subsection{Gradients}

We compute logarithmic index and velocity dispersion gradients performing a linear $\chi^{2}$ fit to the data points within $a<a_{\mathrm{e}}$, where $a$ is the distance from the center along the major axis.

$\Delta($ Index $)=\frac{\Delta \log (\text { Index })}{\Delta \log \left(a / a_{\mathrm{e}}\right)}$.

The fitted gradients and the logarithmic values of the indices and $\sigma$ at $1 a_{\mathrm{e}}$ are listed in Tables A.2 and A.3, respectively. The number distribution of the gradients and their median values are shown in Fig. 2. The velocity dispersion $\sigma$ and the metallic line indices $\mathrm{Mg} b$ and $\langle\mathrm{Fe}\rangle$ show on average negative gradients. The median of the Balmer line gradient, instead, is positive. The $\sigma$-gradients measured here (with a median values of -0.063 ) agree quite well with those found by Davies et al. (1987) and are slightly steeper than the modeling of Jørgensen et al. (1995), who use -0.04 . $\mathrm{Mg} b$ gradients are not available in the literature, the $\mathrm{Mg}_{2}$ gradient measured by Davies et al. (1993; $\left.\triangle \mathrm{Mg}_{2}=-0.059 \pm 0.022\right)$, however, tends to be slightly steeper than our values ( -0.037 for $\log \mathrm{Mg} b$, see Fig. 2). The median $\langle\mathrm{Fe}\rangle$ gradients in Fig. 2 are also somewhat shallower than measurements from Davies et al. (1993) and Gorgas et al. (1990), but are consistent within the errors. Reliable $\mathrm{H} \beta$ gradients for meaningful comparisons are not available in the literature.

Interestingly, we do not find any statistically significant correlation between index gradients and their central values or central velocity dispersion. There is only a very weak hint, that galaxies with stronger central metallic lines have steeper index gradients. Similar results for the $\mathrm{Mg}_{2}$ index have been found for 114 and 42 elliptical galaxies in the field by González \& Gorgas (1995) and Carollo et al. (1993), respectively.

We do find a correlation, however, between the metallic index gradients and the gradients in velocity dispersion. In Fig. 3 it is shown that both $\Delta \mathrm{Mg} b$ and $\Delta\langle\mathrm{Fe}\rangle$ become steeper with a steepening of $\Delta \sigma$ at the significance level of $\approx 3 \sigma$ and $5 \sigma$, respectively. Hence we basically confirm the validity of the assumption, made by Jørgensen et al. (1995) when modeling aperture corrections, that the $\operatorname{Mg} b$ and $\langle\mathrm{Fe}\rangle$ gradients correlate with the gradient of $\sigma$. However, this does not hold for $\mathrm{H} \beta$ (see 3c), where the median gradient is slightly positive. Moreover, our results indicate an aperture correction slightly steeper (but consistent within the errors) than the one proposed by Jørgensen et al. (1995) for the $\sigma$ measurements $\left(\sigma_{\text {ap }} / \sigma_{\text {norm }}=\left(r_{\text {ap }} / r_{\text {norm }}\right)^{-0.06}\right.$ instead of $\left(\sigma_{\text {ap }} / \sigma_{\text {norm }}=\left(r_{\text {ap }} / r_{\text {norm }}\right)^{-0.04}\right)$, a shallower one for the $\langle\mathrm{Fe}\rangle$ values $\left(\langle\mathrm{Fe}\rangle_{\mathrm{ap}} /\langle\mathrm{Fe}\rangle_{\text {norm }}=\left(r_{\mathrm{ap}} / r_{\text {norm }}\right)^{-0.03}\right)$, while on the spot for 


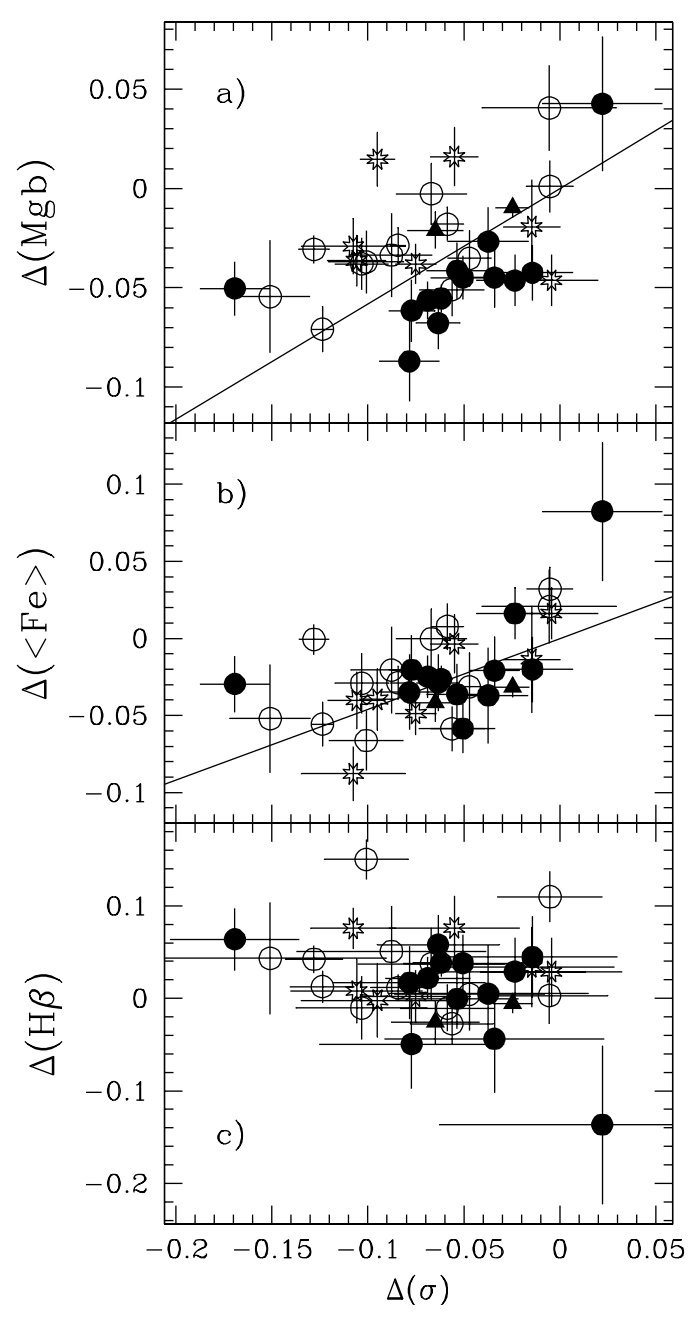

Fig. 3. The fitted logarithmic gradient of the $\operatorname{Mg}_{b}(\mathbf{a}),<\mathrm{Fe}>$ (b) and $\mathrm{H} \beta$ (c) index versus the fitted logarithmic gradient of the velocity dispersion $\sigma$. Symbols as in Fig. 1. The lines show the relations $\langle\Delta \mathrm{Mg} b\rangle \approx 0.58 \times\langle\Delta \sigma\rangle$ and $\langle\Delta\langle\mathrm{Fe}\rangle\rangle \approx 0.46 \times\langle\Delta \sigma\rangle$ (see Fig. 2).

the $\operatorname{Mg} b$ data $\left(\operatorname{Mg} b_{\text {ap }} / \operatorname{Mg} b_{\text {norm }}=\left(r_{\text {ap }} / r_{\text {norm }}\right)^{-0.04}\right)$. Anyway, in a recent paper Moore et al. (2002) show that the aperture correction is usually negligible since it is typically smaller or at most of the order of the individual index errors. It is also interesting to note that the slope of the local $\triangle \mathrm{Mg} b-\Delta \sigma$ correlation is not very different from the one of the global correlation of Eq. (4).

Finally we note that, like for the central index values, no correlation between the index gradients and the density profile of the Coma cluster is present in our galaxy sample.

\section{Ages, metallicities and $[\alpha / \mathrm{Fe}]$ ratios}

\subsection{The model}

In the following we use the stellar population models of TMB in order to derive the stellar population parameters age, metallicity, and $\alpha / \mathrm{Fe}$ ratio from the line indices $\mathrm{H} \beta, \mathrm{Mg} b$, and $\langle\mathrm{Fe}\rangle$.

The TMB models take into account the effects on the Lick indices from element abundance ratio changes, hence give Lick indices of simple stellar populations not only as a function of age and metallicity but also as a function of the $\alpha / \mathrm{Fe}$ ratio. They are based on the evolutionary population synthesis code of Maraston (1998). The impact from element ratio changes is computed with the help of the Tripicco \& Bell (1995) response functions, using an extension of the method introduced by Trager et al. (2000a). Because of the inclusion of element ratio effects, the models allow for the clear distinction between total metallicity $[Z / \mathrm{H}]$ and the $\alpha$ to iron-peak elements ratio $[\alpha / \mathrm{Fe}]$. The latter can be best derived from the classical Lick indices $\mathrm{Mg} b$ and Fe5270 and/or Fe5335 (Maraston et al. 2003, TMB). The models of TMB cover the ages 1 to $15 \mathrm{Gyr}$, metallicities $-2.25 \leq[\mathrm{Z} / \mathrm{H}] \leq 0.67$, and the abundance ratios $-0.3 \leq[\alpha / \mathrm{Fe}] \leq 0.5$.

The Balmer line $\mathrm{H} \beta$ is widely used as an age indicator to break the age-metallicity degeneracy (González 1993; Worthey 1994), because it is very sensitive to warm turnoff stars. We note the caveat, however, that the possible presence of unusually warm horizontal branch stars in the stellar population can hamper the usefulness of Balmer lines as age indicators and seriously affect the age diagnostics (Maraston \& Thomas 2000). Such blue horizontal branch stars may originate from either a metal-poor subpopulation or metal-rich populations with blue horizontal branch morphologies due to enhanced mass loss along the red giant branch evolutionary phase. The TMB models are constructed with the canonical mass loss rates along the red giant branch (Fusi-Pecci \& Renzini 1990), that have been calibrated on galactic globular clusters (Maraston \& Thomas 2000; Maraston et al. 2003). The effect described above, i.e. anomalies in the horizontal branch morphology as a further parameter besides age, metallicity, and $\alpha / \mathrm{Fe}$ ratio, is not considered in the present paper.

We derive the three stellar population parameters age, metallicity, and $\alpha / \mathrm{Fe}$ ratio from the three line indices $\mathrm{H} \beta, \mathrm{Mg} b$, and $\langle\mathrm{Fe}\rangle$ in a twofold iterative procedure. First, we arbitrarily fix the $\alpha / \mathrm{Fe}$ ratio, and determine ages and metallicities for the index pairs $(\mathrm{H} \beta, \mathrm{Mg} b)$ and $(\mathrm{H} \beta,\langle\mathrm{Fe}\rangle)$, by starting with arbitrary age-metallicity pairs, which we modify iteratively until both index pairs are reproduced. The two metallicities obtained from $\mathrm{Mg} b$ and $\langle\mathrm{Fe}\rangle$, respectively, are used to adjust the $\alpha / \mathrm{Fe}$ ratio, and to start a new iteration. These steps are repeated until the age-metallity pairs derived from $(\mathrm{H} \beta, \mathrm{Mg} b)$ and $(\mathrm{H} \beta,\langle\mathrm{Fe}\rangle)$ at a given $\alpha / \mathrm{Fe}$ ratio are consistent within 1 per cent accuracy. For ages and metallicities between the grid points quoted above, we interpolate linearly.

\subsection{Central values}

From the central line indices (Table A.1) we now derive the central ages, metallicities and $\alpha / \mathrm{Fe}$ ratios of the stellar population for the early type galaxies investigated in this paper using the TMB stellar population model described in Sect. 4.1.

\subsubsection{The diagnostic diagrams}

The left diagram in Fig. 4 shows the distribution of the central $\mathrm{H} \beta$ and $[\mathrm{MgFe}]^{\prime}$ indices plotted with the stellar population models of TMB. In this parameter space, mean age and total 

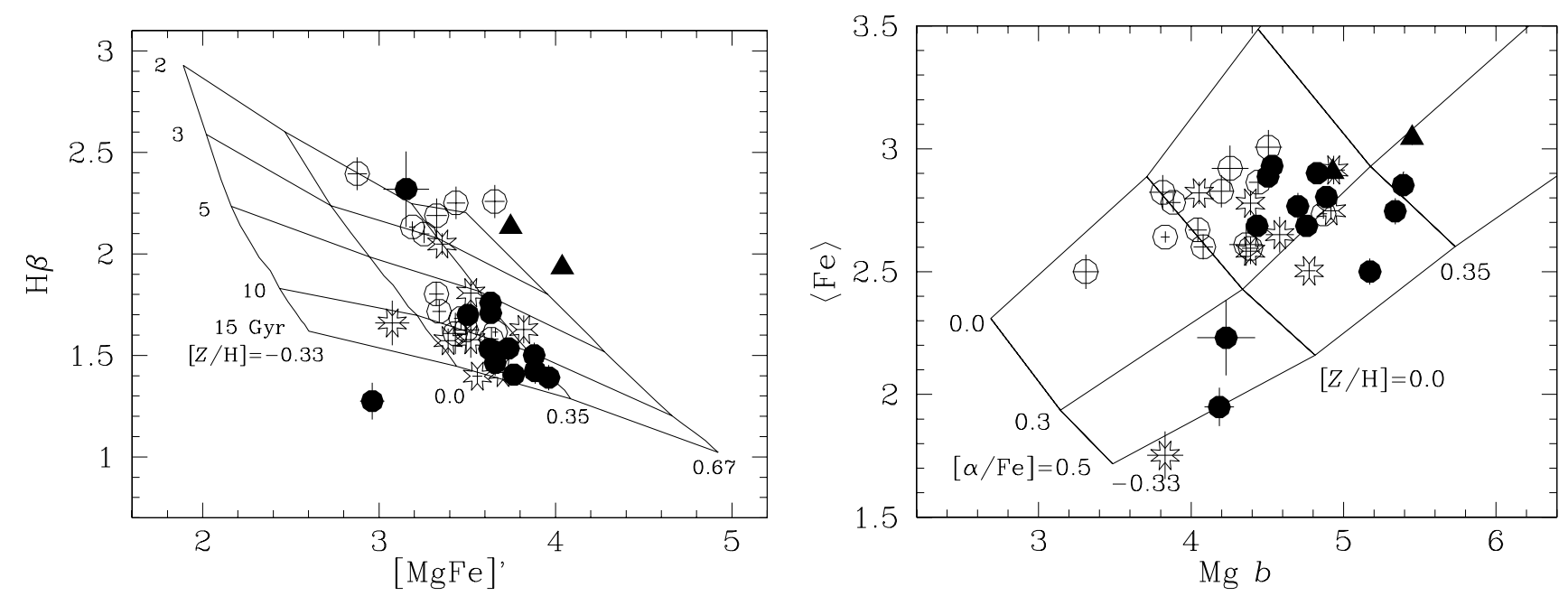

Fig. 4. The distribution of the central values of $\mathrm{H} \beta$ and $[\mathrm{MgFe}]^{\prime}$ indices (left panel) and $\langle\mathrm{Fe}\rangle$ and $\mathrm{Mg} b$ indices (right panel) averaged over $0.1 a_{\mathrm{e}}$ of the 35 early type galaxies of the Coma cluster sample. Symbols as in Fig. 1. The lines indicate models (TMB) of constant ages and metallicities (see labels). Models in the right panel are for fixed age (12 Gyr) and various $\alpha / \mathrm{Fe}$ ratios (see labels).

Table 1. Type dependent mean values and $1 \sigma \mathrm{rms}$ scatter of the central ages, metallicities $([Z / \mathrm{H}])$ and $[\alpha / \mathrm{Fe}]$ ratios.

\begin{tabular}{cccccccc}
\hline \hline Type & $N$ & $\begin{array}{c}<\text { age }> \\
{[\mathrm{Gyr}]}\end{array}$ & $\begin{array}{c}\text { rms (age) } \\
{[\mathrm{Gyr}]}\end{array}$ & $\langle[\mathrm{Z} / \mathrm{H}]\rangle$ & $\operatorname{rms}([\mathrm{Z} / \mathrm{H}])$ & $\langle[\alpha / \mathrm{Fe}]\rangle$ & $\operatorname{rms}([\alpha / \mathrm{Fe}])$ \\
\hline $\mathrm{E}$ & 11 & 10.5 & 3.1 & 0.24 & 0.06 & 0.26 & 0.06 \\
$\mathrm{E} / \mathrm{S} 0$ & 7 & 11.2 & 4.4 & 0.12 & 0.17 & 0.27 & 0.04 \\
$\mathrm{~S}_{1}$ & 7 & 9.6 & 1.3 & 0.14 & 0.06 & 0.21 & 0.06 \\
$\mathrm{~S}_{2}$ & 5 & 2.6 & 0.6 & 0.34 & 0.16 & 0.18 & 0.05 \\
\hline
\end{tabular}

metallicity can be best read-off by eye, as both indices are practically insensitive to $\alpha / \mathrm{Fe}$ ratio variations as shown in TMB. In the right-hand panel of Fig. 4 we show models and data in the $\mathrm{Mg} b-\langle\mathrm{Fe}\rangle$ plane, which provides a reasonably good first approximation of $\alpha / \mathrm{Fe}$ ratios, although age effects cannot be neglected for the final derivation of $\alpha / \mathrm{Fe}$ ratios. The models in the right panel are plotted for fixed age $(12 \mathrm{Gyr})$. The precise central ages, metallicities and $\alpha / \mathrm{Fe}$ ratios derived for our sample are listed in Table B.1.

Most objects have super-solar total metallicities and $\alpha / \mathrm{Fe}$ element ratios, hence they are $\alpha / \mathrm{Fe}$ enhanced. The sample spans a relatively large range in average ages from 2 to 15 Gyr in agreement with a recent study of Coma cluster galaxies by Trager et al. (2003). The bimodal distribution in the $[\mathrm{MgFe}]^{\prime}-\mathrm{H} \beta$ parameter space is particularly interesting. We notice one "clump" at old ages scattering about an average age of $10 \mathrm{Gyr}$ with a rather narrow distribution in metallicities $(0 \leq[Z / \mathrm{H}] \leq 0.35)$, and a second one at roughly $2 \mathrm{Gyr}$ spanning a larger range in metallicities $(0 \leq[Z / \mathrm{H}] \leq 0.8)$. There is a very clear separation between the two. While the "old clump" contains all types of objects, i.e. ellipticals (E), lenticulars (S0) and transition types (E/S0), the "young clump" is by far dominated by $\mathrm{S} 0$ galaxies plus the two cDs. This result was already indicated by the distribution of the $\mathrm{H} \beta$ indices discussed in Sect. 3.1, where a subclass of lenticulars with high $\mathrm{H} \beta$ was pointed out.

The average stellar parameters of these subclasses are summarized in Table 1. The averages exclude, however, the galaxy GMP 3958 (E) because of its extremely low Balmer index $(\mathrm{H} \beta=1.29$, see Table A.1), which implies an extrapolated unreasonable age of $24 \mathrm{Gyr}$ (see Fig. 4). Also the three objects GMP3329 (cD), GMP2921 (cD), and GMP3561 (S0) are not included in these average values, because they lie outside the model grid, so that their derived stellar parameters are strongly affected by uncertainties caused by extrapolation. Finally, we excluded galaxy GMP3201 from the further discussion (averages in Table 1 and linear fits in Figs. 5 and 6), since the errors of the measured indices and hence the corresponding stellar parameters are systematically larger than those of all other objects.

Table 1 confirms the S0 dichotomy found by Poggianti et al. (2001a), who investigated a sample of 52 Coma early type galaxies. They interpret the young class $\left(\mathrm{SO}_{2}\right)$ as being the descendants of typical star-forming spirals whose star formation has been stopped due to the dense cluster environment, while the evolutionary history of the $\mathrm{S}_{1}$ class and Es should be identical. The low average ages of the young S0 subclass $\left(\mathrm{SO}_{2}\right)$ measured would then be the direct consequence of recent star 
formation that had ceased just recently due to the infall in the dense cluster environment. The resulting extended star formation histories would imply, however, a significant enrichment of iron from Type Ia supernovae, so that low $\alpha / \mathrm{Fe}$ ratios should be observed in contrast to the results found here (see Table 1). The relatively high $\alpha / \mathrm{Fe}$ ratios disfavor the occurence of recent star formation. They would be more compatible with the alternative interpretation that the high Balmer line indices measured in the objects of class $\mathrm{SO}_{2}$ are actually caused by the presence of unusually blue horizontal branches in these objects rather than by young stellar populations (see Sect. 4.1).

\subsubsection{Correlations with velocity dispersion}

Figure 5 shows the derived stellar parameters as a function of velocity dispersion $\sigma$. The parameter which correlates best with $\sigma$ is the element ratio $\alpha / \mathrm{Fe}$ (panel c). Excluding the class of young SOs and cDs, also total metallicity correlates reasonably well with velocity dispersion (panel b). Age, instead, does not seem to be a major parameter among early-type galaxies (see also Trager et al. 2000b; Kuntschner et al. 2002; Trager et al. 2003). Again, the separation in two classes, the "young" one being dominated by S0 galaxies, is very striking (panel a).

It should be emphasized, however, that the age derivation is most affected by observational errors. A proper analysis to decide about possible trends of age with galaxy mass therefore requires a larger galaxy sample and the consideration of errors, e.g., via Monte Carlo simulations. Such an analysis is carried out in Thomas et al. (2003b) for a sample of 126 field and cluster galaxies (including the present sample). Interestingly, the dichotomy mentioned above is confirmed for this larger sample (including galaxies in low density environments), and the data of the "old clump" are best consistent with age slightly increasing with galaxy mass. Still, the above conclusion that age is only a secondary parameter remains. The existence of a $[\mathrm{Z} / \mathrm{H}]-\sigma$ correlation, instead, is further reinforced by the study of Thomas et al. (2003b).

Figure 5 shows that the elliptical galaxies and the "old" lenticular galaxies (class $\mathrm{S}_{1}$ in Table 1) follow the same correlations of metallicity and $\alpha / \mathrm{Fe}$ ratio with velocity dispersion. The total metallicities of the young lenticulars (class $\mathrm{SO}_{2}$ in Table 1$)$, instead, are significantly $(\sim 0.4 \mathrm{dex})$ higher than they are expected to be for their $\sigma$, while their $\alpha / \mathrm{Fe}$ ratios are consistent with their velocity dispersions.

Using the mean correlations shown in Fig. 5 (assuming a constant age of $9 \mathrm{Gyr}$ ) we can now reproduce the $\mathrm{Mg} b$ and $\langle\mathrm{Fe}\rangle$ indices with the TMB models, and in this way estimate the relative importance of metallicity and $\alpha / \mathrm{Fe}$ variations in generating the global $\mathrm{Mg}-\sigma$ and $\mathrm{Fe}-\sigma$ relations. The resulting index $\sigma$ relations for models in which only metallicity or only $\alpha / \mathrm{Fe}$ increases with $\sigma$ are shown in Fig. 1 by the long and short-dashed lines, respectively. We deduce the contributions from metallicity and $\alpha / \mathrm{Fe}$ variations to the $\mathrm{Mg}-\sigma$ relation to be approximately 70 and 30 per cent, respectively. Hence, we confirm that metallicity is the main driver of the relation, as suggested in the past. We show, however, that the $\alpha / \mathrm{Fe}$ ratio does play a non-negligible role. Figure 1 further illustrates

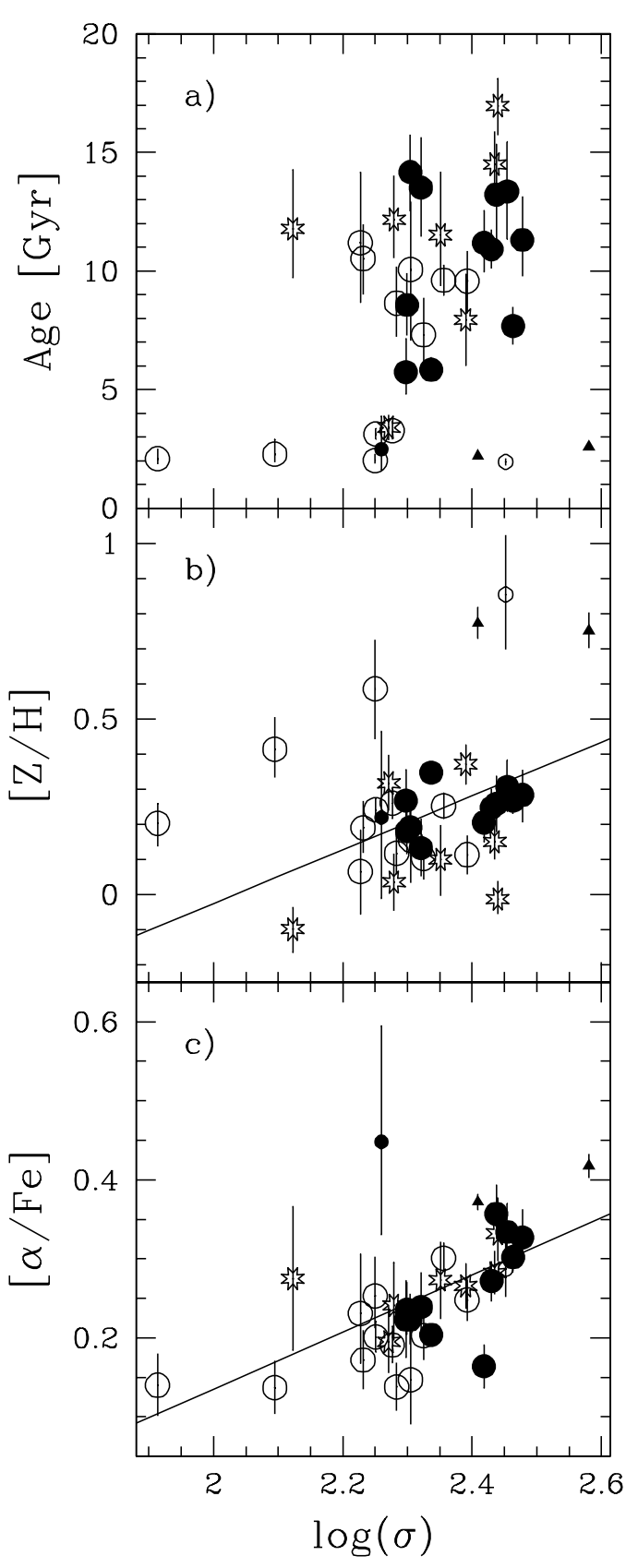

Fig. 5. Central ages, metallicities $([\mathrm{Z} / \mathrm{H}])$ and the $[\alpha / \mathrm{Fe}]$ ratios listed in Table B.1 plotted versus the central velocity dispersion $\sigma$. Symbols as in Fig. 1. Straight lines indicate the linear least square fits for panels b) (excluding the low-age, high metallicity objects) and c) (excluding the Objects GMP 3329, 2921, 3561 and 3201, small symbols, see Sect. 4.2.1), giving the following relations: $[\mathrm{Z} / \mathrm{H}]=-1.56( \pm 0.27)+$ $0.77( \pm 0.10) \times \log (\sigma),[\alpha / \mathrm{Fe}]=-0.59( \pm 0.21)+0.36( \pm 0.09) \times \log (\sigma)$.

that the shallower $\mathrm{Fe}-\sigma$ is the result of the combination of the (positive) metallicity dependence with the negative $\alpha / \mathrm{Fe}$ (anti-) correlation.

\subsubsection{Correlations between the stellar population parameters}

Possible correlations between the three stellar population parameters are explored in Fig. 6. There is a weak indication 


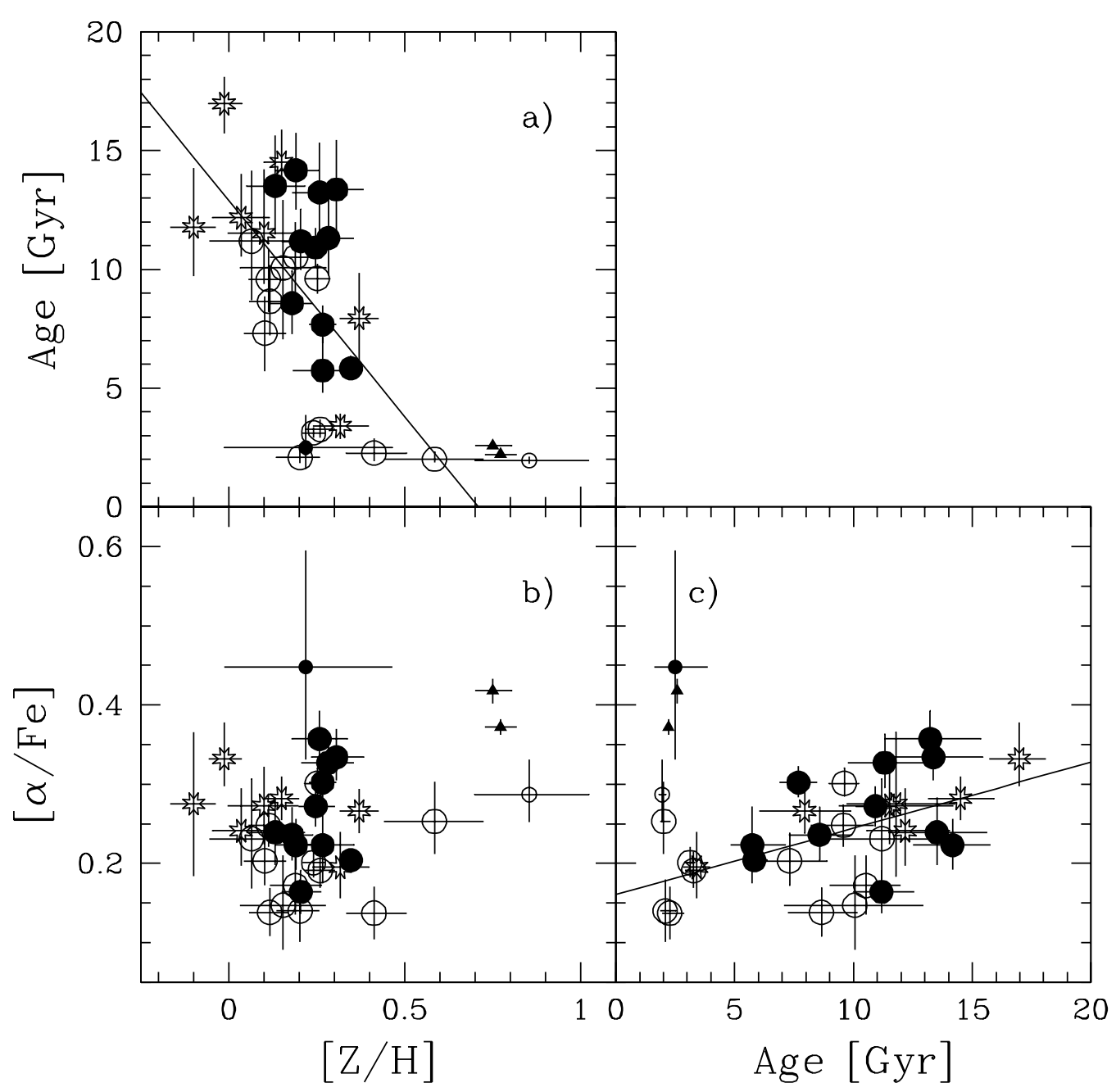

Fig. 6. Central ages, metallicities $([Z / H])$ and the $[\alpha / \mathrm{Fe}]$ ratios listed in Table B.1 plotted versus each other. Symbols as in Fig. 1. The linear least square fits for panel a) and c) give following relations a) age $=12.9( \pm 1.2)-18.2( \pm 4.7) \times[Z / \mathrm{H}]$ and $\mathbf{c})[\alpha / \mathrm{Fe}]=0.16( \pm 0.02)+0.008( \pm 0.002) \times$ age and are indicated by the straight lines. Object GMP 3329, 2921, 3561 and 3201 (small symbols) were not included in the fit (see Sect. 4.2.1).

for a possible age-metallicity anti-correlation among the old subclass (above $\sim 5$ Gyr). It is more likely, however, that an anti-correlation at such low significance is an artifact caused by correlated errors in age and metallicity (Trager et al. 2000b; Kuntschner et al. 2002; Thomas et al. 2003b). Note also that the young subclass is not restricted to high metallicities, but exhibits, instead, a large range in metallicities from $[\mathrm{Z} / \mathrm{H}]=$ 0.2 to 0.9 dex.

We detect the trend that galaxies with higher $\alpha / \mathrm{Fe}$ ratios tend to have older average ages (panel c, see also Thomas et al. 2002). This result is important, as it supports the connection between $\alpha / \mathrm{Fe}$ ratio and formation timescale. It is important to take into account that correlated errors actually lead to the opposite trend, namely higher $\alpha / \mathrm{Fe}$ ratios at younger ages (Thomas et al. 2003b).

It does not come as a surprise that, like the central indices, also the central ages, metallicities and $[\alpha / \mathrm{Fe}]$ ratios derived for our galaxy sample do not correlate with the density profile of the Coma cluster. Note that, except for a very small fraction of young field elliptical galaxies (see also Kuntschner et al. 2001), also Thomas et al. (2002) could not find any significant differences between early-type galaxies in low and high density environments with respect to their stellar parameters. Environmental density seems indeed to be only of secondary importance for the formation of early-type galaxies.

\subsection{Gradients}

We determine the gradients in ages, metallicities, and $\alpha / \mathrm{Fe}$ ratios from the fitted index gradients $(\mathrm{H} \beta, \mathrm{Mg} b,\langle\mathrm{Fe}\rangle)$ and the logarithmic fit values at $1 a_{\mathrm{e}}$ given in Tables A. 2 and A. 3 by means of the SSP models of TMB. The ages, metallicities, and $\alpha / \mathrm{Fe}$ ratios at $0.1 a_{\mathrm{e}}$ and $1 a_{\mathrm{e}}$ are derived and the gradient was set as the difference between the two. Hence, we obtain the variation of the stellar population parameters per decade within $1 a_{\mathrm{e}}$ (see also Eq. (5)). The 1- $\sigma$ errors were evaluated through Monte Carlo simulations taking into account the errors in the gradients and the logarithmic fit values of the three indices. For the two objects GMP3661 and GMP4679 no reliable gradients could be obtained, because the very high $\mathrm{H} \beta$ indices 


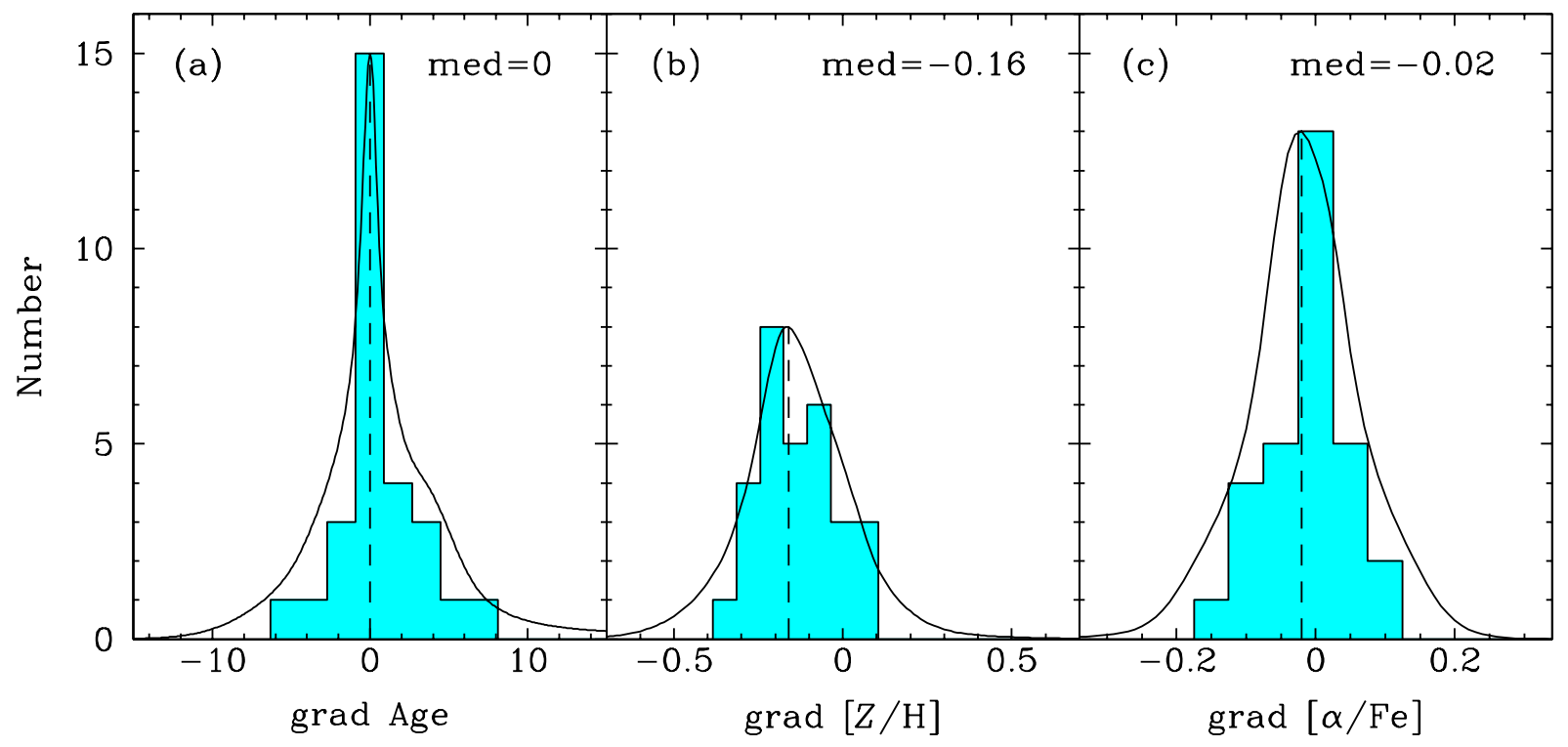

Fig. 7. Distributions of the gradients derived for age (Gyr, panel a)), metallicity (panel b)), and $\alpha / \mathrm{Fe}$ ratio (panel c)). The median values are given in the panels, respectively. The solid lines are obtained by summing up the distributions of the gradients folded with their Gaussian errors.

at $1 a_{\mathrm{e}}$ would require extrapolation to ages much younger than covered by the grid of the present SSP models, which makes a reliable age determination impossible. Like in case of the central values, the very low $\mathrm{H} \beta$ of GMP3958 again made its age determination unreliable.

The final gradients of the age, metallicity and the $[\alpha / \mathrm{Fe}]$ ratio including their errors are listed in Table B.2. Histograms of their number distributions and the median values are shown in Fig. 7. The solid lines are obtained by summing up the distributions of the gradients folded with their Gaussian errors.

\subsubsection{Age and $\alpha / \mathrm{Fe}$ ratio}

The most striking result is that, on average, the early-type galaxies in the Coma cluster do not exhibit gradients in age or $\alpha / \mathrm{Fe}$ ratio. The number distributions of the age and $\alpha / \mathrm{Fe}$ ratio gradients (panels a and $\mathrm{c}$ in Fig. 7) show both a very clear peak at zero. As the distributions in age and $\alpha / \mathrm{Fe}$ ratio are not well-defined Gaussians, we approximate their rms (Gaussian widths $\sigma$ ) by the deviation of 68 per cent of the objects from the median value of the distribution. The resulting values for the age and $\alpha / \mathrm{Fe}$ gradients are $2.7 \mathrm{Gyr}$ and $0.05 \mathrm{dex}$, respectively, and fully consistent with the respective median errors of the gradients (2.9 Gyr and 0.05 dex, see Table B.2). In both cases, 91 per cent of the objects fall inside $2 \sigma$ of the distributions. To conclude, the non-detection of a gradient within one effective radius in both age and $\alpha / \mathrm{Fe}$ for the sample studied here is a statistically significant result. Devations from the median value can be explained by observational errors alone. It is worth noting that we do not find a correlation between $\operatorname{grad}$ Age and grad $[\alpha / \mathrm{Fe}]$, or between $\operatorname{grad} \mathrm{Z}$ and $\operatorname{grad}[\alpha / \mathrm{Fe}]$, which further supports the above conclusion. There is a weak anticorrelation between grad Age and $\operatorname{grad} \mathrm{Z}$ as a consequence of the expected correlated errors.
The lack of gradients in age and $\alpha / \mathrm{Fe}$ ratio is particularly interesting as both parameters reflect formation timescales. The presence of $\alpha / \mathrm{Fe}$ enhanced stellar populations is obviously not restricted to galaxy centers. Early-type galaxies are globally $\alpha / \mathrm{Fe}$ enhanced, at least within their effective radii. Hence, the formation epochs and timescales of the stellar populations do not significantly change within the galaxy as a function of radius. This conclusion goes along well with the lack of a significant age gradient. The stellar populations in a galaxy may form on long or on short timescales, but they form simultaneously in the entire galaxy independent of their distance to the galaxy center. Note that also the lack of a significant evolution with redshift of color gradients in early-type galaxies strongly disfavors the presence of age gradients in early-type galaxies (Saglia et al. 2000; Tamura et al. 2000).

This implies that scenarios with strong inside-out or outside-in formation processes are both disfavored. No significant residual star formation can have occurred either in the outskirts or in the center of a galaxy. This stands in conflict with simple monolithic models, that predict the onset of galactic winds to occur first at large radii, so that a positive gradient in $\alpha / \mathrm{Fe}$ should be detected (Martinelli et al. 1998; Thomas et al. 1999).

On the other hand, current models of hierarchical galaxy formation cannot easily accommodate globally $\alpha / \mathrm{Fe}$ enhanced stellar populations (Thomas \& Kauffmann 1999). In general, one expects significant star formation to rapidly occur in the galaxy center triggered by a merger event (Schweizer 1990; Barnes 1992; Bender \& Surma 1992). Therefore an $\alpha / F e$ overabundant stellar population should be present there (i.e. a centrally localized negative gradient, Thomas 1999). However, note that the spatial resolution of our data (typically $\sim 2^{\prime \prime}$, see Mehlert et al. 2000) allows us to detect decoupled cores in only two of our 35 galaxies (see Mehlert et al. 1998) and that our $\alpha / \mathrm{Fe}$ gradients avoid the central regions. 

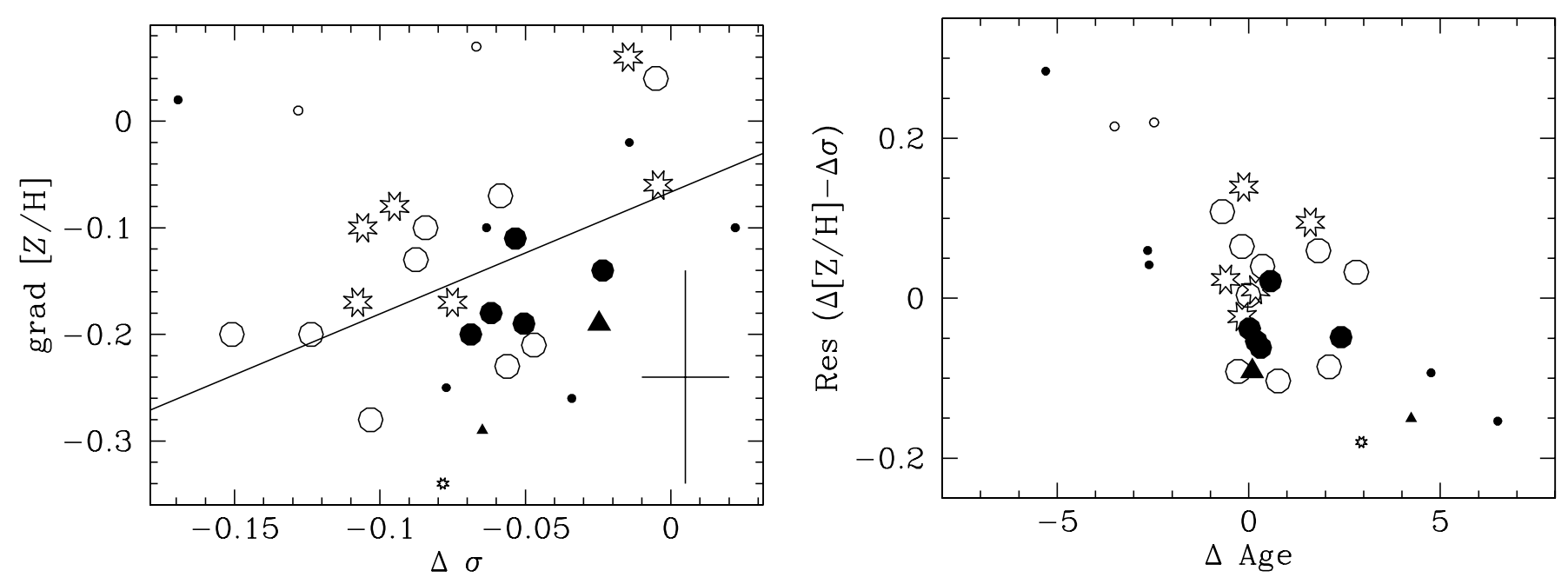

Fig. 8. Left panel: metallicity gradient as a function of the logarithmic gradient in velocity dispersion. Symbols as in Fig. 1. Galaxies outside one $\sigma$ about the mean of the age gradients' distribution (see Fig. 7) are plotted with smaller symbols. The typical error is given in the lower right-hand corner. Right panel: residuals from the correlation shown in the left panel by the solid line as a function of the age gradient.

\subsubsection{Metallicity}

While age and the $\alpha /$ Fe ratio indicate zero gradients, we do find a significant negative median metallicity gradient (panel b in Fig. 7). The distribution exhibits a well-defined peak at $\triangle[Z / \mathrm{H}] / \triangle \log a_{\mathrm{e}}=-0.16$. The rms of this peak is $0.12 \mathrm{dex}$ and fully consistent with the median error in the metallicity gradient ( $\sim 0.1$ dex, see Table B.2). More than 97 per cent of the data points fall inside $2 \sigma$ around the peak of the distribution. The median metallicity gradient found here is in agreement, although a bit flatter than previous determinations (e.g., Davies et al. 1993). It has already been discussed extensively in the literature (e.g., Peletier et al. 1990; see also references in the Introduction) that galaxy formation in a simple dissipative collapse (e.g., Larson 1974) would produce average metallicity gradients of about 0.5 dex per decade (Carlberg 1984), well in excess of the slope derived here. Hence some kind of mixing process, for instance induced by galaxy mergers, are required to flatten out the metallicity gradient (White 1980).

The total metallicity of a stellar population only depends on the efficiency of star formation, i.e. the fraction of gas turned into stars (e.g., Tinsley 1980). It seems therefore natural to assume that a relationship between metallicity and escape velocity produces the metallicity gradient (Franx \& Illingworth 1990; Davies et al. 1993; Martinelli et al. 1998). In the galaxies' center, where the gravitational potential is highest, star formation and hence the enrichment of metals is more effective than in outer regions with lower gravitational potential.

We find empirical support for this picture, as our data indicate a correlation between the metallicity gradient and the gradient in velocity dispersion (left panel in Fig. 8). Galaxies with steeper metallicity gradients have also steeper gradients in velocity dispersion. Note that the determination of the metallicity gradient is affected by uncertainties in the $\mathrm{H} \beta$ index gradient and hence the age gradient, due to correlated errors caused by the age-metallicity degeneracy. In other words, an erroneously steep negative age gradient causes a too flat or even positive metallicity gradient and vice versa. Therefore we determine the linear fit (solid line in left panel of Fig. 8) for those objects that are inside $1 \sigma$ about the mean of the age gradients' distribution (see Fig. 7). The galaxies outside one $\sigma$, hence objects with unusually steep positive or negative age gradients, are shown as smaller symbols. It can be seen that most outliers fall in that latter category. The right panel of Fig. 8 illustrates this effect esplicitly, by showing the residuals from the correlation between the gradients in metallicity and velocity dispersion (solid line) as a function of the age gradient. Objects with age gradients close to the median value follow the correlation surprisingly well, given the large error bars.

Finally we note that we did not find any significant evidence for correlations of the stellar population gradients with central velocity dispersion or other central stellar population properties, as well as other galaxy properties like galaxy type, velocity dispersion, and environmental density in terms of radial distance from the cluster center.

\subsubsection{Global vs. intrinsic $\mathrm{Mg}-\sigma$ relations}

In Sect. 4.2 we have shown that the variations of the $\alpha / \mathrm{Fe}$ ratio drive $30 \%$ of the global $\mathrm{Mg}-\sigma$ relation. The intrinsic $\mathrm{Mg}-\sigma$ relation, i.e. local velocity dispersion versus local $\mathrm{Mg}$ linestrength inside a galaxy, instead, must be due to metallicity variations alone, because of the absence of a significant gradient in $\alpha / \mathrm{Fe}$ and age. In other words, the global and intrinsic $\mathrm{Mg}-\sigma$ relations do not have a common origin. Somewhat amazingly, however, the slopes of the global and local relations are not very different. 


\section{Conclusions}

From high $S / N$ spectra of 35 early type galaxies in the Coma cluster we have measured central values and gradients within one effective radius of velocity dispersion and the line indices $\mathrm{H} \beta, \mathrm{Mg} b, \mathrm{Fe} 5270$, and Fe5335. With the new $\alpha / \mathrm{Fe}$ sensitive stellar population models of TMB, we have determined central values and gradients of the stellar population parameters age, total metallicity, and $\alpha / \mathrm{Fe}$ element ratio.

Central values We find a very clear age dichotomy among the population of S0 galaxies in the Coma cluster. The first class is dominated by old ( $\sim 10 \mathrm{Gyr})$ stellar populations and is practically indistinguishable from the elliptical galaxy population. The lenticulars of the second class, that also includes the two cD galaxies, have very young ( 2 Gyr) average ages, hence are dominated by recent major star formation episodes. They may be the descendants of typical star-forming spirals whose star formation has been stopped due to the dense cluster environment (Poggianti et al. 2001a).

The element ratio $\alpha / \mathrm{Fe}$ correlates with central velocity dispersion, and drives $30 \%$ of the $\mathrm{Mg}-\sigma$ relation. We find the tendency that older galaxies have higher $\alpha / \mathrm{Fe}$ ratios (see also Thomas et al. 2002), which implies that the most massive galaxies in the Coma cluster had not only the shortest star formation timescales (because of the $\alpha / \mathrm{Fe}$ ratio; e.g., Matteucci 1994; Thomas et al. 1999) but were also the first to form.

Interestingly, none of the indices or the stellar population parameters depend on the density profile of the Coma cluster, which spans a range of about 3 dex. We conclude that environmental effects did not significantly influence on the evolution of the early-type galaxies in the Coma cluster.

Gradients The most striking result from studying the stellar population gradients (within $1 R_{e}$ ) of our sample is the absence of a gradient in both age and $\alpha / \mathrm{Fe}$ ratio. The first direct important inference is that the existence of $\alpha / \mathrm{Fe}$ enhanced stellar populations in early-type galaxies is not restricted to their centers but it is a global phenomenon. The star formation timescales do not vary significantly inside a galaxy as a function of radius. The local potential at a given radius does not affect the formation timescales.

It does affect, instead, the fraction of gas lost in a galactic wind, as we detect a significant negative metallicity gradient. At larger radii, the potential is shallower, i.e. the escape velocity is lower, so that star formation is less complete and the metallicity is lower than in the center (e.g., Davies et al. 1993). This conclusion is further supported by the fact that we find a relationship between the metallicity gradient and the gradient in velocity dispersion.

Finally, the presence of a metallicity gradient and the absence of gradients in age and $\alpha / \mathrm{Fe}$ ratio show that the intrinsic $\mathrm{Mg}-\sigma$ relation, i.e. local velocity dispersion versus $l o-$ cal $\mathrm{Mg}$ line-strength inside a galaxy, is driven by metallicity alone. We have shown that the global relation, instead, is driven by both metallicity and $\alpha / \mathrm{Fe}$ ratio, the former contributing roughly 70 the latter 30 per cent. Hence, the global and the intrinsic $\mathrm{Mg}-\sigma$ relations have different origins, the former being at least partially produced by formation timescales ( $\alpha / \mathrm{Fe}$ ratio), the latter by the local gravitational potential (metallicity) alone.

Acknowledgements. We thank the referee Bianca Poggianti for very helpful comments and Scott Trager for the many interesting discussions. This work was supported by the Deutsche Forschungsgemeinschaft via project Be 1091/6 and SFB 375.

\section{Appendix B: Stellar parameters}

Table B.1. The central ages, metallicities $([\mathrm{Z} / \mathrm{H}])$ and the $[\alpha / \mathrm{Fe}]$ ratios derived from the indices listed in Table A.1 combined with the models from TMB (see Fig. 4 as well as Sect. 4.1).

\begin{tabular}{|c|c|c|c|c|c|c|}
\hline GMP Nr. & $\begin{array}{r}\text { age } \\
{[\mathrm{Gyr}]}\end{array}$ & $\begin{array}{r}\text { dage } \\
{[\mathrm{Gyr}]}\end{array}$ & {$[\mathrm{Z} / \mathrm{H}]$} & $\mathrm{d}[\mathrm{Z} / \mathrm{H}]$ & {$[\alpha / \mathrm{Fe}]$} & $\mathrm{d}[\alpha / \mathrm{Fe}]$ \\
\hline 3329 & 2.2 & 0.1 & 0.77 & 0.04 & 0.37 & 0.01 \\
\hline 2921 & 2.6 & 0.2 & 0.75 & 0.05 & 0.42 & 0.02 \\
\hline 4928 & 14.5 & 1.4 & 0.15 & 0.05 & 0.28 & 0.03 \\
\hline 4822 & 11.2 & 1.3 & 0.21 & 0.06 & 0.16 & 0.03 \\
\hline 1750 & 11.3 & 1.7 & 0.28 & 0.08 & 0.33 & 0.03 \\
\hline 2390 & 13.2 & 2.0 & 0.26 & 0.08 & 0.36 & 0.03 \\
\hline 2795 & 9.6 & 1.3 & 0.11 & 0.06 & 0.25 & 0.03 \\
\hline 3792 & 13.4 & 2.1 & 0.31 & 0.07 & 0.33 & 0.03 \\
\hline 2629 & 8.7 & 1.5 & 0.12 & 0.07 & 0.14 & 0.03 \\
\hline 3561 & 2.0 & 0.15 & 0.854 & 0.163 & 0.287 & 0.04 \\
\hline 2000 & 8.6 & 1.3 & 0.18 & 0.06 & 0.24 & 0.03 \\
\hline 2413 & 7.3 & 1.6 & 0.10 & 0.06 & 0.21 & 0.03 \\
\hline 4829 & 7.9 & 1.9 & 0.37 & 0.06 & 0.27 & 0.03 \\
\hline 3510 & 14.2 & 1.6 & 0.19 & 0.06 & 0.22 & 0.03 \\
\hline 2417 & 11.5 & 2.4 & 0.10 & 0.10 & 0.27 & 0.05 \\
\hline 2440 & 13.5 & 2.1 & 0.13 & 0.08 & 0.24 & 0.04 \\
\hline 3414 & 11.2 & 2.7 & 0.07 & 0.12 & 0.23 & 0.07 \\
\hline 4315 & 12.2 & 1.7 & 0.04 & 0.08 & 0.24 & 0.05 \\
\hline 3073 & 10.5 & 1.5 & 0.19 & 0.07 & 0.17 & 0.04 \\
\hline 1853 & 10.1 & 2.9 & 0.16 & 0.12 & 0.15 & 0.06 \\
\hline 3201 & 2.5 & 1.1 & 0.22 & 0.24 & 0.45 & 0.13 \\
\hline 3661 & 2.0 & 0.2 & 0.59 & 0.14 & 0.25 & 0.05 \\
\hline 4679 & 2.1 & 0.3 & 0.20 & 0.06 & 0.14 & 0.04 \\
\hline 3352 & 3.4 & 0.5 & 0.32 & 0.08 & 0.20 & 0.04 \\
\hline 2535 & 2.3 & 0.5 & 0.41 & 0.09 & 0.14 & 0.03 \\
\hline 3958 & - & - & - & - & - & - \\
\hline 2776 & 11.8 & 2.3 & -0.10 & 0.06 & 0.28 & 0.09 \\
\hline 0144 & 5.8 & 0.5 & 0.34 & 0.02 & 0.20 & 0.01 \\
\hline 0282 & 7.7 & 0.8 & 0.26 & 0.04 & 0.30 & 0.02 \\
\hline 0756 & 3.1 & 0.2 & 0.24 & 0.03 & 0.20 & 0.02 \\
\hline 1176 & 3.3 & 0.4 & 0.26 & 0.04 & 0.19 & 0.02 \\
\hline 1990 & 17.0 & 1.2 & -0.01 & 0.05 & 0.33 & 0.04 \\
\hline 5279 & 10.9 & 0.8 & 0.25 & 0.05 & 0.27 & 0.03 \\
\hline 5568 & 9.6 & 0.6 & 0.25 & 0.04 & 0.30 & 0.02 \\
\hline 5975 & 5.7 & 1.2 & 0.27 & 0.09 & 0.22 & 0.05 \\
\hline
\end{tabular}


Table B.2. Gradients of the ages, metallicities $([\mathrm{Z} / \mathrm{H}])$ and $[\alpha / \mathrm{Fe}])$ ratios derived from the fitted gradients values at $1 a_{\mathrm{e}}$ listed in Tables A.2 and A.3 combined with the models from TMB (see Sect. 4.3 for details.)

\begin{tabular}{|c|c|c|c|c|c|c|}
\hline $\begin{array}{c}\text { GMP } \\
\mathrm{Nr} \text {. }\end{array}$ & $\begin{array}{l}\triangle \text { age } \\
{[\mathrm{Gyr}]}\end{array}$ & $\begin{array}{l}\mathrm{d} \triangle \mathrm{age} \\
{[\mathrm{Gyr}]}\end{array}$ & $\Delta[\mathrm{Z} / \mathrm{H}]$ & $\mathrm{d} \triangle[\mathrm{Z} / \mathrm{H}]$ & $\Delta[\alpha / \mathrm{Fe}]$ & $\mathrm{d} \triangle[\alpha / \mathrm{Fe}]$ \\
\hline 3329 & 0.1 & 0.3 & -0.19 & 0.07 & 0.02 & 0.02 \\
\hline 2921 & 4.2 & 1.3 & -0.29 & 0.12 & -0.02 & 0.03 \\
\hline 4928 & 0.2 & 3.4 & -0.06 & 0.10 & -0.16 & 0.04 \\
\hline 4822 & 2.4 & 3.2 & -0.14 & 0.09 & -0.01 & 0.04 \\
\hline 1750 & 4.8 & 5.2 & -0.25 & 0.13 & -0.09 & 0.07 \\
\hline 2390 & 6.5 & 5.8 & -0.26 & 0.14 & 0.02 & 0.06 \\
\hline 2795 & -2.5 & 1.4 & 0.01 & 0.05 & -0.05 & 0.02 \\
\hline 3792 & -2.6 & 4.8 & -0.02 & 0.12 & -0.01 & 0.06 \\
\hline 2629 & -0.7 & 3.2 & 0.04 & 0.29 & 0.03 & 0.08 \\
\hline 3561 & -0.3 & 0.8 & -0.28 & 0.19 & 0.04 & 0.06 \\
\hline 2000 & 0.6 & 2.5 & -0.11 & 0.10 & -0.01 & 0.06 \\
\hline 2413 & 1.8 & 2.2 & -0.07 & 0.07 & -0.10 & 0.04 \\
\hline 4829 & -0.2 & 2.5 & -0.17 & 0.08 & 0.03 & 0.04 \\
\hline 3510 & 0.3 & 2.5 & -0.19 & 0.08 & 0.04 & 0.04 \\
\hline 2417 & -7.3 & 2.9 & 0.21 & 0.07 & 0.09 & 0.05 \\
\hline 2440 & -5.3 & 2.9 & 0.02 & 0.10 & -0.02 & 0.04 \\
\hline 3414 & 2.8 & 4.4 & -0.13 & 0.14 & -0.03 & 0.09 \\
\hline 4315 & 0.1 & 3.0 & -0.10 & 0.07 & 0.04 & 0.04 \\
\hline 3073 & -3.5 & 3.7 & 0.07 & 0.11 & 0.04 & 0.06 \\
\hline 1853 & 0.4 & 4.2 & -0.20 & 0.18 & -0.01 & 0.09 \\
\hline 3201 & 17.9 & 7.5 & -0.10 & 0.30 & -0.10 & 0.14 \\
\hline 3661 & - & - & - & - & - & - \\
\hline 4679 & - & - & - & - & - & - \\
\hline 3352 & -0.6 & 1.1 & -0.17 & 0.11 & 0.12 & 0.04 \\
\hline 2535 & 0.8 & 0.8 & -0.23 & 0.12 & -0.06 & 0.05 \\
\hline 3958 & - & - & - & - & - & - \\
\hline 2776 & -0.1 & 2.5 & 0.06 & 0.13 & -0.02 & 0.09 \\
\hline 0144 & 0.0 & 1.2 & -0.18 & 0.04 & -0.02 & 0.02 \\
\hline 0282 & 0.2 & 2.0 & -0.20 & 0.07 & -0.06 & 0.03 \\
\hline 0756 & -0.0 & 0.6 & -0.20 & 0.06 & -0.02 & 0.05 \\
\hline 1176 & -0.2 & 0.4 & -0.10 & 0.05 & -0.02 & 0.03 \\
\hline 1990 & 1.6 & 4.0 & -0.08 & 0.07 & 0.12 & 0.06 \\
\hline 5279 & -2.6 & 3.5 & -0.10 & 0.11 & -0.05 & 0.06 \\
\hline 5568 & 2.1 & 3.4 & -0.21 & 0.12 & 0.00 & 0.06 \\
\hline 5975 & 2.9 & 2.4 & -0.34 & 0.16 & -0.11 & 0.07 \\
\hline
\end{tabular}

\section{References}

Barnes, J. 1992, ApJ, 393, 484 Baugh, C. M., Cole, S., \& Frenk, C. 1996, MNRAS, 283, 1361 Bender, R., \& Surma, P. 1992, A\&A, 258, 250

Bender, R., Burstein, D., \& Faber, S. M. 1993, ApJ, 411, 153 Bender, R., Saglia, R. P., Ziegler, B., et al. 1998, ApJ, 493, 529 Bower, R. G., Lucey, J. R., \& Ellis, R. S. 1992, MNRAS, 254, 601 Briel, U., Henry, J. P., \& Böhringer, H. 1992, A\&A, 259, L31
Burstein, D., Davies, R. L., Dressler, A., et al. 1988, in Towards Understanding Galaxies at Large Redshifts, ed. R. K. Kron, \& A. Renzini (Dordrecht: Kluwer)

Carlberg, R. G. 1984, ApJ, 286, 403

Carter, D., Mobasher, B., Bridges, T. J., et al. 2002, ApJ, 567, 772

Carollo, M., Danziger, I. J., \& Buson, L. 1993, MNRAS, 265, 553

Cole, S., Lacey, C. G., Baugh, C. M., \& Frenk, C. S. 2000, MNRAS, 319, 168

Colless, M., Burstein, D., Davies, R. L., et al. 1999, MNRAS, 303, 813

Davies, R. L., Sadler, E. M., \& Peletier, R. F. 1993, MNRAS, 262, 650

Davies, R. L., Burstein, D., Dressler, A., et al. 1987, ApJS, 64, 581

Djorgovski, S., \& Davis, M. 1987, ApJ, 313, 59

Dressler, A., Lynden-Bell, D., Burstein, D., et al. 1987, ApJ, 313, 42

Eggen, O. J., Lynden-Bell, D., \& Sandage, A. R. 1962, ApJ, 136, 748

Faber, S. M. 1977, in The Evolution of Galaxies and Stellar Populations, ed. B. Tinsley, \& R. B. Larson (New Haven: Yale Univ. Obs.), 157

Franx, M., \& Illingworth, G. D. 1990, ApJ, 359, L41

Fusi-Pecci, F., \& Renzini, A. 1976, A\&A, 46, 447

González, J. J., \& Gorgas, J. 1996, in Fresh views of elliptical galaxies, ASP, 86, ed. A. Buzzoni, A. Renzini, \& A. Serrano, 225

González, J. J. 1993, Ph.D. Thesis, University of California, Santa Cruz

Gorgas, J., Efstathiou, G., \& Aragón-Salamanca, A. 1990, MNRAS, 245,217

Jimenez, R., Friaca, A. C. S., Dunlop, J. S., et al. 1999, MNRAS, 305, L16

Jørgensen, I. 1999, MNRAS, 306, 607

Jørgensen, I., Franx, M., \& Kjærgaard, P. 1995, MNRAS, 276, 1341

Kauffmann, G. 1996, MNRAS, 281, 487

Kobayashi, C., \& Arimoto, N. 1999, ApJ, 527, 573

Kuntschner, H., \& Davies, R. L. 1998, MNRAS, 295, L29

Kuntschner, H., MNRAS, 315, 184

Kuntschner, H., Lucey, J. R., Smith, R. J., Hudson, M. J., \& Davies, R. L. 2001, MNRAS, 323, 615

Kuntschner, H., Smith, R. J., Colless, M., et al. 2002, MNRAS, 337, 172

Lacey, C., \& Cole, S. 1993, MNRAS, 262, 627

Larson, R. B. 1974, MNRAS, 166, 385

Maraston, C. 1998, MNRAS, 300, 872

Maraston, C., \& Thomas, D. 2000, ApJ, 541, 126

Maraston, C., Greggio, L., Renzini, A., et al. 2003, A\&A, 400, 823

Martinelli, A., Matteucci, F., \& Colafrancesco, S. 1998, MNRAS, 298, 42

Mehlert, D., Saglia, R. P., Bender, R., et al. 2000, A\&AS, 141, 449 (Paper I)

Mehlert, D. 1998, Ph.D. Thesis, Universität München

Mehlert, D., Saglia, R. P., Bender, R., \& Wegner, G. 1998, A\&A, 332, 33

Moore, S. A. W., Lucey, J. R., Kuntschner, H., et al. 2002, MNRAS, 336, 382

Peletier, R. F., Davies, R. L., Illingworth, G. D., Davis, L. E., \& Cawson, M. 1990, AJ, 100, 1091

Poggianti, B. M., Bridges, T. J., Carter, D., et al. 2001a, ApJ, 563, 118

Poggianti, B. M., Bridges, T. J., Mobasher, et al. 2001b, ApJ, 562, 689

Saglia, R. P., Maraston, C., Greggio, L., Bender, R., \& Ziegler, B. 2000, A\&A, 360, 911

Schweizer, F. 1990, in Dynamics and Interactions of Galaxies, ed. R. Wielen (Springer - Verlag), 60

Tamura, N., Kobayashi, C., Arimoto, N., Kodama, T., \& Ohta, K. 2000, AJ, 119, 2134

Terlevich, A. I., \& Forbes, D. A. 2002, MNRAS, 330, 547 
Tinsley, B. 1980, Fundam. Cosmic Phys., 5, 287

Thomas, D. 1999, MNRAS, 306, 655

Thomas, D., \& Kauffmann, G. 1999, in Spectrophotometric dating of stars and galaxies, ed. I. Hubeny, S. Heap, \& R. Cornett, ASP Conf. Ser., 192, 261

Thomas, D., Greggio, L., \& Bender, R. 1999, MNRAS, 302, 537

Thomas, D., Maraston, C., \& Bender, R. 2002, Ap\&SS, 281, 371

Thomas, D., Maraston, C., \& Bender, R. 2003a, MNRAS, 339, 897 (TMB)

Thomas, D., Maraston, C., \& Bender, R. 2003b, in preparation

Trager, S. C., Worthey, G., Faber, S. M., et al. 1998, ApJS, 116, 1

Trager, S. C., Faber, S. M., Worthey, G., \& González, J. J. 2000a, AJ, 119,164

Trager, S. C., Faber, S. M., Worthey, G., \& González, J. J. 2000b, AJ, 120,165
Trager, S. C., Faber, S. M., \& Dressler, A. 2003, ApJ, in preparation Treu, T., Stiavelli, M., Casertano, S., Moller, P., \& Bertin, G. 1999, MNRAS, 308, 1037

Tripicco, M. J., \& Bell, R. A. 1995, AJ, 110, 3035

van Dokkum, P. G., \& Franx, M. 1996, MNRAS, 281, 985

van Dokkum, P. G., Franx, M., Kelson, D. D., \& Illingworth, G. D. 2001, ApJ, 553, L39

van Dokkum, P. G., \& Stanford, S. A. 2003, ApJ, 585, 78

Vazdekis, A., Peletier, R. F., Beckman, J. E., \& Casuso, E. 1996, ApJS, 106, 307

Wegner, G., Corsini, E. M., Saglia, R. P., et al. 2002, A\&A, 395, 753

White, S. D. M. 1980, MNRAS, 191, 1

Worthey, G. 1994, ApJS, 95, 107 
D. Mehlert et al.: Spectroscopy of Coma early-type galaxies. III. Gradients, Online Material p 1

\section{Online Material}


D. Mehlert et al.: Spectroscopy of Coma early-type galaxies. III. Gradients, Online Material p 2

\section{Appendix A: Line indices}

Table A.1. The central values (averaged over $0.1 a_{\mathrm{e}}$ ) of the indices $\mathrm{H}_{\beta}, \mathrm{Mg}_{b}, \mathrm{Fe}_{5270}, \mathrm{Fe}_{5335}$ and of the velocity dispersion $\sigma$.

\begin{tabular}{|c|c|c|c|c|c|c|c|c|c|c|c|c|}
\hline $\mathrm{Nr}$. & $\begin{array}{c}\text { GMP } \\
\text { Nr. }\end{array}$ & Type & $\begin{array}{l}\mathrm{H} \beta \\
{[\AA]}\end{array}$ & $\begin{array}{l}\mathrm{dH} \beta \\
{[\AA]}\end{array}$ & $\begin{array}{c}\operatorname{Mg} b \\
{[\AA]}\end{array}$ & $\begin{array}{c}\mathrm{dMg} b \\
{[\AA ̊]}\end{array}$ & $\begin{array}{c}\text { Fe5270 } \\
{[\AA]}\end{array}$ & $\begin{array}{c}\mathrm{dFe} 5270 \\
{[\AA]}\end{array}$ & $\begin{array}{c}\text { Fe5335 } \\
{[\AA ̊]}\end{array}$ & $\begin{array}{c}\mathrm{dFe} 5335 \\
{[\AA ̊]}\end{array}$ & $\begin{array}{c}\langle\sigma\rangle \\
{\left[\mathrm{km} \mathrm{s}^{-1}\right]}\end{array}$ & $\begin{array}{c}\mathrm{d}\langle\sigma\rangle \\
{\left[\mathrm{km} \mathrm{s}^{-1}\right]}\end{array}$ \\
\hline 1 & 3329 & $\mathrm{D}$ & 2.13 & 0.03 & 4.91 & 0.03 & 2.82 & 0.04 & 2.93 & 0.04 & 256.5 & 1.5 \\
\hline 2 & 2921 & D & 1.94 & 0.07 & 5.45 & 0.08 & 2.93 & 0.08 & 3.15 & 0.09 & 380.6 & 5.1 \\
\hline 3 & 4928 & $\mathrm{E} / \mathrm{S} 0$ & 1.41 & 0.10 & 4.90 & 0.11 & 2.83 & 0.12 & 2.68 & 0.13 & 272.3 & 4.7 \\
\hline 4 & 4822 & E & 1.61 & 0.10 & 4.57 & 0.11 & 2.91 & 0.12 & 2.94 & 0.14 & 262.0 & 3.2 \\
\hline 5 & 1750 & E & 1.55 & 0.13 & 5.17 & 0.13 & 3.08 & 0.15 & 2.47 & 0.17 & 300.5 & 2.7 \\
\hline 6 & 2390 & $\mathrm{E}$ & 1.43 & 0.13 & 5.33 & 0.14 & 2.93 & 0.15 & 2.57 & 0.17 & 273.8 & 2.7 \\
\hline 7 & 2795 & S0 & 1.60 & 0.09 & 4.33 & 0.096 & 2.90 & 0.104 & 2.30 & 0.12 & 246.8 & 2.2 \\
\hline 8 & 3792 & E & 1.39 & 0.13 & 5.39 & 0.14 & 2.99 & 0.15 & 2.72 & 0.17 & 284.7 & 4.9 \\
\hline 9 & 2629 & So & 1.72 & 0.13 & 4.32 & 0.13 & 2.78 & 0.12 & 2.72 & 0.13 & 191.9 & 2.0 \\
\hline 10 & 3561 & So & 2.26 & 0.14 & 4.48 & 0.12 & 2.96 & 0.16 & 2.99 & 0.17 & 283.0 & 4.3 \\
\hline 11 & 2000 & E & 1.70 & 0.12 & 4.43 & 0.13 & 2.87 & 0.15 & 2.50 & 0.16 & 199.1 & 2.2 \\
\hline 12 & 2413 & So & 1.77 & 0.11 & 4.11 & 0.09 & 2.85 & 0.14 & 2.35 & 0.14 & 211.3 & 2.0 \\
\hline 13 & 4829 & E/S0 & 1.63 & 0.10 & 4.94 & 0.12 & 3.01 & 0.12 & 2.81 & 0.13 & 245.5 & 2.9 \\
\hline 14 & 3510 & $\mathrm{E}$ & 1.41 & 0.12 & 4.83 & 0.13 & 2.98 & 0.15 & 2.82 & 0.16 & 201.5 & 2.8 \\
\hline 15 & 2417 & S0/E & 1.61 & 0.17 & 4.55 & 0.18 & 2.82 & 0.20 & 2.39 & 0.22 & 224.4 & 3.8 \\
\hline 16 & 2440 & E & 1.46 & 0.15 & 4.70 & 0.17 & 2.95 & 0.19 & 2.58 & 0.21 & 209.4 & 3.7 \\
\hline 17 & 3414 & So & 1.61 & 0.22 & 4.36 & 0.23 & 2.82 & 0.27 & 2.41 & 0.29 & 168.7 & 9.5 \\
\hline 18 & 4315 & E/S0 & 1.57 & 0.15 & 4.39 & 0.16 & 2.67 & 0.19 & 2.50 & 0.21 & 190.2 & 4.1 \\
\hline 19 & 3073 & So & 1.58 & 0.14 & 4.44 & 0.15 & 3.12 & 0.16 & 2.60 & 0.18 & 170.4 & 2.4 \\
\hline 20 & 1853 & So & 1.65 & 0.22 & 4.33 & 0.24 & 2.91 & 0.25 & 2.75 & 0.28 & 201.8 & 3.8 \\
\hline 21 & 3201 & E & 2.29 & 0.42 & 4.12 & 0.44 & 2.53 & 0.48 & 1.88 & 0.56 & 181.7 & 5.7 \\
\hline 22 & 3661 & So & 2.25 & 0.16 & 4.20 & 0.17 & 2.79 & 0.18 & 2.86 & 0.20 & 177.8 & 7.3 \\
\hline 23 & 4679 & So & 2.37 & 0.12 & 3.42 & 0.13 & 2.45 & 0.14 & 2.62 & 0.16 & 82.0 & 2.2 \\
\hline 24 & 3352 & $\mathrm{E} / \mathrm{S} 0$ & 2.05 & 0.17 & 4.00 & 0.18 & 2.80 & 0.19 & 2.73 & 0.22 & 186.7 & 6.7 \\
\hline 25 & 2535 & S0 & 2.22 & 0.08 & 3.93 & 0.09 & 2.91 & 0.09 & 2.88 & 0.10 & 124.4 & 3.0 \\
\hline 26 & 3958 & $\mathrm{E}$ & 1.29 & 0.14 & 4.18 & 0.15 & 2.29 & 0.16 & 1.59 & 0.18 & 152.9 & 2.1 \\
\hline 27 & 2776 & S0/E & 2.00 & 0.2 & 4.21 & 0.22 & 2.69 & 0.24 & 1.90 & 0.27 & 132.6 & 2.8 \\
\hline 28 & 0144 & E & 1.76 & 0.04 & 4.53 & 0.04 & 2.89 & 0.05 & 2.98 & 0.05 & 217.1 & 1.4 \\
\hline 29 & 0282 & $\mathrm{E}$ & 1.71 & 0.07 & 4.75 & 0.08 & 2.88 & 0.09 & 2.51 & 0.10 & 290.6 & 2.3 \\
\hline 30 & 0756 & S0 & 2.15 & 0.06 & 3.83 & 0.07 & 2.70 & 0.08 & 2.55 & 0.09 & 178.3 & 0.7 \\
\hline 31 & 1176 & So & 2.13 & 0.07 & 3.87 & 0.08 & 2.80 & 0.09 & 2.55 & 0.10 & 189.0 & 0.8 \\
\hline 32 & 1990 & E/S0 & 1.40 & 0.04 & 4.78 & 0.12 & 2.83 & 0.13 & 2.19 & 0.15 & 275.1 & 3.7 \\
\hline 33 & 5279 & $\mathrm{E}$ & 1.54 & 0.093 & 4.89 & 0.10 & 2.91 & 0.12 & 2.70 & 0.13 & 269.1 & 2.7 \\
\hline 34 & 5568 & S0 & 1.62 & 0.066 & 4.82 & 0.07 & 2.80 & 0.08 & 2.63 & 0.09 & 226.9 & 1.8 \\
\hline 35 & 5975 & $\mathrm{E}$ & 1.81 & 0.18 & 4.39 & 0.20 & 2.88 & 0.23 & 2.68 & 0.25 & 198.4 & 5.4 \\
\hline
\end{tabular}


D. Mehlert et al.: Spectroscopy of Coma early-type galaxies. III. Gradients, Online Material p 3

Table A.2. The fitted logarithmic gradients inside $a<1 a_{\mathrm{e}}$ of the indices $\mathrm{H}_{\beta}, \mathrm{Mg}_{b}, \mathrm{Fe}_{5270}, \mathrm{Fe}_{5335}$ and the velocity dispersion $\sigma$.

\begin{tabular}{|c|c|c|c|c|c|c|c|c|c|c|}
\hline GMP Nr. & $\Delta \mathrm{H}_{\beta}$ & $\mathrm{d} \triangle \mathrm{H}_{\beta}$ & $\Delta \mathrm{Mg}_{b}$ & $\mathrm{~d} \triangle \mathrm{Mg}_{b}$ & $\Delta \mathrm{Fe}_{5270}$ & $\mathrm{~d} \triangle \mathrm{Fe}_{5270}$ & $\Delta \mathrm{Fe}_{5335}$ & $\mathrm{~d} \triangle \mathrm{Fe}_{5335}$ & $\Delta \sigma$ & $\mathrm{d} \triangle \sigma$ \\
\hline 3329 & -0.006 & 0.010 & -0.010 & 0.005 & -0.035 & 0.009 & -0.006 & 0.009 & -0.025 & 0.009 \\
\hline 2921 & -0.026 & 0.023 & -0.021 & 0.009 & -0.036 & 0.017 & -0.045 & 0.018 & -0.065 & 0.015 \\
\hline 4928 & 0.029 & 0.037 & -0.046 & 0.013 & -0.026 & 0.024 & 0.064 & 0.023 & -0.004 & 0.024 \\
\hline 4822 & 0.029 & 0.037 & -0.046 & 0.013 & -0.026 & 0.024 & 0.064 & 0.023 & -0.023 & 0.020 \\
\hline 1750 & -0.050 & 0.048 & -0.062 & 0.016 & -0.046 & 0.028 & 0.025 & 0.036 & -0.077 & 0.012 \\
\hline 2390 & -0.044 & 0.057 & -0.045 & 0.015 & -0.019 & 0.027 & -0.022 & 0.035 & -0.034 & 0.013 \\
\hline 2795 & 0.042 & 0.015 & -0.031 & 0.007 & -0.002 & 0.011 & 0.004 & 0.018 & -0.128 & 0.008 \\
\hline 3792 & 0.045 & 0.044 & -0.042 & 0.014 & -0.034 & 0.027 & 0.007 & 0.033 & -0.014 & 0.021 \\
\hline 2629 & 0.110 & 0.027 & 0.001 & 0.013 & -0.002 & 0.019 & 0.082 & 0.020 & -0.005 & 0.012 \\
\hline 3561 & -0.010 & 0.034 & -0.038 & 0.013 & -0.017 & 0.027 & -0.042 & 0.029 & -0.103 & 0.014 \\
\hline 2000 & -0.001 & 0.032 & -0.041 & 0.014 & -0.026 & 0.024 & -0.039 & 0.032 & -0.053 & 0.016 \\
\hline 2413 & -0.011 & 0.025 & -0.018 & 0.008 & 0.016 & 0.018 & 0.006 & 0.026 & -0.059 & 0.008 \\
\hline 4829 & 0.002 & 0.029 & -0.038 & 0.010 & -0.037 & 0.018 & -0.056 & 0.021 & -0.075 & 0.011 \\
\hline 3510 & 0.038 & 0.031 & -0.045 & 0.011 & -0.050 & 0.021 & -0.056 & 0.025 & -0.050 & 0.017 \\
\hline 2417 & 0.076 & 0.034 & 0.016 & 0.015 & 0.015 & 0.022 & -0.031 & 0.030 & -0.055 & 0.013 \\
\hline 2440 & 0.064 & 0.034 & -0.050 & 0.014 & -0.046 & 0.024 & -0.005 & 0.028 & -0.169 & 0.018 \\
\hline 3414 & 0.051 & 0.049 & -0.034 & 0.021 & 0.018 & 0.034 & -0.063 & 0.048 & -0.088 & 0.021 \\
\hline 4315 & 0.008 & 0.035 & -0.036 & 0.013 & -0.024 & 0.024 & -0.056 & 0.029 & -0.106 & 0.015 \\
\hline 3073 & 0.037 & 0.039 & -0.003 & 0.015 & 0.009 & 0.024 & -0.008 & 0.032 & -0.067 & 0.019 \\
\hline 1853 & 0.043 & 0.060 & -0.054 & 0.028 & -0.044 & 0.045 & -0.058 & 0.055 & -0.151 & 0.021 \\
\hline 3201 & -0.136 & 0.085 & 0.043 & 0.034 & 0.011 & 0.058 & 0.165 & 0.070 & 0.022 & 0.031 \\
\hline 3661 & 0.150 & 0.022 & -0.037 & 0.016 & -0.069 & 0.027 & -0.047 & 0.027 & -0.101 & 0.020 \\
\hline 4679 & 0.003 & 0.031 & 0.040 & 0.022 & -0.000 & 0.034 & 0.045 & 0.033 & -0.005 & 0.035 \\
\hline 3352 & 0.076 & 0.022 & -0.029 & 0.014 & -0.026 & 0.021 & -0.094 & 0.029 & -0.108 & 0.027 \\
\hline 2535 & -0.027 & 0.022 & -0.051 & 0.013 & -0.069 & 0.019 & -0.044 & 0.021 & -0.056 & 0.017 \\
\hline 3958 & 0.005 & 0.052 & -0.027 & 0.017 & -0.028 & 0.034 & -0.046 & 0.058 & -0.037 & 0.021 \\
\hline 2776 & 0.034 & 0.043 & -0.019 & 0.024 & -0.058 & 0.042 & 0.053 & 0.058 & -0.015 & 0.015 \\
\hline 0144 & 0.038 & 0.015 & -0.055 & 0.007 & -0.019 & 0.012 & -0.031 & 0.013 & -0.062 & 0.006 \\
\hline 0282 & 0.022 & 0.022 & -0.056 & 0.010 & -0.042 & 0.018 & -0.003 & 0.022 & -0.069 & 0.006 \\
\hline 0756 & 0.013 & 0.017 & -0.071 & 0.011 & -0.054 & 0.019 & -0.053 & 0.022 & -0.124 & 0.006 \\
\hline 1176 & 0.012 & 0.014 & -0.029 & 0.009 & -0.028 & 0.014 & -0.019 & 0.016 & -0.084 & 0.004 \\
\hline 1990 & -0.002 & 0.040 & 0.015 & 0.014 & -0.036 & 0.024 & 0.087 & 0.030 & -0.095 & 0.009 \\
\hline 5279 & 0.058 & 0.032 & -0.068 & 0.013 & -0.056 & 0.025 & 0.012 & 0.027 & -0.063 & 0.011 \\
\hline 5568 & 0.005 & 0.039 & -0.035 & 0.014 & -0.046 & 0.029 & -0.012 & 0.032 & -0.047 & 0.012 \\
\hline 5975 & 0.017 & 0.040 & -0.087 & 0.020 & -0.029 & 0.033 & -0.023 & 0.037 & -0.078 & 0.016 \\
\hline
\end{tabular}


D. Mehlert et al.: Spectroscopy of Coma early-type galaxies. III. Gradients, Online Material p 4

Table A.3. Logarithmic fit values at $1 a_{\mathrm{e}}$ of the indices $\mathrm{H}_{\beta}, \mathrm{Mg}_{b}, \mathrm{Fe}_{5270}, \mathrm{Fe}_{5335}$ and the velocity dispersion $\sigma$.

\begin{tabular}{|c|c|c|c|c|c|c|c|c|c|c|}
\hline GMP Nr. & $\begin{array}{c}\log _{a_{\mathrm{e}}} \\
\mathrm{H}_{\beta}\end{array}$ & $\begin{array}{c}\operatorname{dlog}_{a_{\mathrm{e}}} \\
\mathrm{H}_{\beta}\end{array}$ & $\begin{array}{l}\log _{a_{\mathrm{e}}} \\
\mathrm{Mg}_{b}\end{array}$ & $\begin{array}{c}\operatorname{dlog}_{a_{\mathrm{e}}} \\
\mathrm{Mg}_{b}\end{array}$ & $\begin{array}{c}\log _{a_{\mathrm{e}}} \\
\mathrm{Fe}_{5270}\end{array}$ & $\begin{array}{l}\mathrm{dlog}_{a_{\mathrm{e}}} \\
\mathrm{Fe}_{5270}\end{array}$ & $\begin{array}{c}\log _{a_{\mathrm{e}}} \\
\mathrm{Fe}_{5335}\end{array}$ & $\begin{array}{l}\operatorname{dlog}_{a_{\mathrm{e}}} \\
\mathrm{Fe}_{5335}\end{array}$ & $\begin{array}{c}\log _{a_{\mathrm{e}}} \\
\sigma\end{array}$ & $\begin{array}{c}\operatorname{dlog}_{a_{\mathrm{e}}} \\
\sigma\end{array}$ \\
\hline 3329 & 0.325 & 0.016 & 0.678 & 0.008 & 0.397 & 0.014 & 0.474 & 0.014 & 2.397 & 0.015 \\
\hline 2921 & 0.246 & 0.030 & 0.708 & 0.012 & 0.409 & 0.022 & 0.426 & 0.024 & 2.477 & 0.020 \\
\hline 4928 & 0.205 & 0.041 & 0.625 & 0.016 & 0.412 & 0.028 & 0.512 & 0.025 & 2.431 & 0.033 \\
\hline 4822 & 0.196 & 0.032 & 0.639 & 0.012 & 0.420 & 0.022 & 0.493 & 0.020 & 2.387 & 0.024 \\
\hline 1750 & 0.114 & 0.049 & 0.636 & 0.016 & 0.433 & 0.028 & 0.446 & 0.033 & 2.379 & 0.012 \\
\hline 2390 & 0.098 & 0.063 & 0.674 & 0.016 & 0.435 & 0.029 & 0.372 & 0.038 & 2.384 & 0.014 \\
\hline 2795 & 0.290 & 0.016 & 0.594 & 0.008 & 0.454 & 0.013 & 0.384 & 0.018 & 2.225 & 0.007 \\
\hline 3792 & 0.198 & 0.033 & 0.680 & 0.011 & 0.445 & 0.022 & 0.461 & 0.025 & 2.434 & 0.020 \\
\hline 2629 & 0.400 & 0.028 & 0.632 & 0.015 & 0.444 & 0.022 & 0.566 & 0.020 & 2.278 & 0.018 \\
\hline 3561 & 0.319 & 0.028 & 0.612 & 0.010 & 0.446 & 0.021 & 0.413 & 0.023 & 2.340 & 0.010 \\
\hline 2000 & 0.232 & 0.028 & 0.599 & 0.013 & 0.425 & 0.022 & 0.357 & 0.029 & 2.229 & 0.016 \\
\hline 2413 & 0.228 & 0.028 & 0.601 & 0.010 & 0.493 & 0.018 & 0.404 & 0.026 & 2.237 & 0.010 \\
\hline 4829 & 0.228 & 0.022 & 0.648 & 0.008 & 0.432 & 0.015 & 0.382 & 0.019 & 2.298 & 0.009 \\
\hline 3510 & 0.202 & 0.026 & 0.625 & 0.010 & 0.406 & 0.019 & 0.370 & 0.024 & 2.245 & 0.015 \\
\hline 2417 & 0.269 & 0.023 & 0.642 & 0.010 & 0.460 & 0.017 & 0.325 & 0.026 & 2.255 & 0.014 \\
\hline 2440 & 0.240 & 0.021 & 0.616 & 0.009 & 0.420 & 0.016 & 0.408 & 0.018 & 2.140 & 0.014 \\
\hline 3414 & 0.258 & 0.033 & 0.611 & 0.015 & 0.461 & 0.023 & 0.326 & 0.034 & 2.116 & 0.024 \\
\hline 4315 & 0.199 & 0.033 & 0.595 & 0.011 & 0.392 & 0.020 & 0.311 & 0.025 & 2.134 & 0.014 \\
\hline 3073 & 0.240 & 0.031 & 0.643 & 0.012 & 0.490 & 0.019 & 0.403 & 0.026 & 2.159 & 0.017 \\
\hline 1853 & 0.263 & 0.044 & 0.582 & 0.023 & 0.423 & 0.036 & 0.394 & 0.045 & 2.132 & 0.016 \\
\hline 3201 & 0.164 & 0.075 & 0.637 & 0.023 & 0.453 & 0.040 & 0.499 & 0.040 & 2.276 & 0.027 \\
\hline 3661 & 0.477 & 0.013 & 0.585 & 0.011 & 0.383 & 0.019 & 0.419 & 0.019 & 2.137 & 0.016 \\
\hline 4679 & 0.383 & 0.029 & 0.590 & 0.020 & 0.391 & 0.033 & 0.481 & 0.031 & 1.903 & 0.036 \\
\hline 3352 & 0.356 & 0.011 & 0.586 & 0.007 & 0.431 & 0.011 & 0.379 & 0.016 & 2.208 & 0.017 \\
\hline 2535 & 0.320 & 0.017 & 0.542 & 0.010 & 0.394 & 0.015 & 0.413 & 0.017 & 2.031 & 0.016 \\
\hline 3958 & 0.118 & 0.031 & 0.599 & 0.011 & 0.337 & 0.021 & 0.161 & 0.036 & 2.149 & 0.014 \\
\hline 2776 & 0.321 & 0.026 & 0.603 & 0.015 & 0.377 & 0.027 & 0.329 & 0.034 & 2.084 & 0.014 \\
\hline 0144 & 0.283 & 0.013 & 0.597 & 0.006 & 0.437 & 0.010 & 0.432 & 0.011 & 2.283 & 0.005 \\
\hline 0282 & 0.254 & 0.017 & 0.610 & 0.008 & 0.412 & 0.014 & 0.400 & 0.016 & 2.366 & 0.006 \\
\hline 0756 & 0.348 & 0.013 & 0.500 & 0.009 & 0.376 & 0.015 & 0.352 & 0.018 & 2.084 & 0.006 \\
\hline 1176 & 0.351 & 0.011 & 0.548 & 0.007 & 0.413 & 0.012 & 0.382 & 0.014 & 2.146 & 0.004 \\
\hline 1990 & 0.127 & 0.023 & 0.668 & 0.007 & 0.412 & 0.016 & 0.433 & 0.018 & 2.267 & 0.011 \\
\hline 5279 & 0.245 & 0.023 & 0.616 & 0.010 & 0.405 & 0.019 & 0.430 & 0.020 & 2.340 & 0.012 \\
\hline 5568 & 0.218 & 0.052 & 0.634 & 0.019 & 0.379 & 0.039 & 0.406 & 0.042 & 2.296 & 0.020 \\
\hline 5975 & 0.269 & 0.022 & 0.572 & 0.012 & 0.432 & 0.018 & 0.420 & 0.021 & 2.215 & 0.013 \\
\hline
\end{tabular}

